\title{
Gênesis da classificação: uma análise de conteúdo a partir da definição
}

\author{
Gercina Ângela de Lima \\ https://orcid.org/0000-0003-0735-3856 \\ I Universidade Federal de Minas Gerais, MG, Brasil. \\ Doutora em Ciência da Informação.
}

\section{http://dx.doi.org/10.1590/1981-5344/32686}

$\mathrm{Na}$ literatura, existem palavras que apresentam mais de um significado nos múltiplos contextos, e isso as faz terem interpretações ambíguas ou mesmo serem consideradas polissêmicas; é o caso da palavra classificação. Nas diversas acepções da palavra, ela pode significar um processo, um sistema de classificação ou uma disciplina. Este artigo tem como objetivo estudar a perspectiva interdisciplinar do significado da palavra classificação, a partir das acepções sugeridas pelos autores da área, com o intuito de traçar sua evolução e contexto histórico. Utiliza-se a base metodológica proposta por Bardin (2011) sobre Análise de conteúdo, aplicada nas 40 definições selecionadas a partir do artigo "Classification" (Hjørland, 2017), como amostra da pesquisa. Os resultados das análises léxicas e categoriais das definições confirmaram os pressupostos de que a classificação traça sua história pelas bases categoriais aristotélicas, tendo começado seu desenvolvimento dicotômico com os estudos de Porfírio, ganhado projeção com o sistema de categorias taxonômicas de Lineu, com a ideia de "divisão e denominação" para classificar os seres em grupos, com a obra A Origem das espécies de Darwin; e, no âmbito da Biblioteconomia e Ciência da Informação, 
com os estudos iniciados pelo bibliotecário Richards (1860-1939), no século XIX, influenciando os estudos de autores como Sayers (1881-1960), Bliss (1870-1955) e. Ranganathan (1892-1972), entre outros. Na área da Biblioteconomia e Ciência da Informação, a classificação tem sido estudada como uma disciplina, um processo de agrupar e ordenar o conhecimento, e como instrumento de representação da informação, contribuindo para a sua organização e recuperação.

Palavras-chave: Classificação. Organização do Conhecimento Biblioteconomia. Ciência da Informação Análise de conteúdo.

\section{Genesis of classification: a content analysis based on the definition}

In the literature, words have more than one meaning in multiple contexts, and this makes them have ambiguous interpretations or even be considered polysemic; this is the case with the word classification. In the different meanings of the word, it can mean a process, a classification system or a discipline. This article studies the interdisciplinary perspective of the meaning of the word classification, from the meanings suggested by the authors of the area, to trace its evolution and historical context. We use the methodological basis proposed by Bardin (2011) on Content Analysis, applied to the 40 definitions selected from the article "Classification" (Hjørland, 2017), as a research sample. The results of lexical and categorical analysis of the definitions confirmed the assumptions that the classification traces its history based on the Aristotelian categorical bases, having started its dichotomous development with the studies of Porphyrios, gaining projection with the system of taxonomic categories of Linnaeus, with the idea of "Division and denomination" to classify beings into groups, with Darwin's Origin of Species; and, in the scope 
of Librarianship and Information Science, with the studies initiated by librarian Richards (1860-1939), in the 19th century, influencing the studies of authors such as Sayers (1881-1960), Bliss (1870-1955) e. Ranganathan (18921972), among others. In the area of Librarianship and Information Science, classification has been studied as a discipline, a process of grouping and ordering knowledge, and as an instrument of information representation, contributing to its organization and recovery.

Keywords: Classification. Knowledge Organization. Librarianship. Information Science. Content analysis.

Recebido em 25.03.2021 Aceito em 31.03.2021

\section{Introdução}

Existem na literatura palavras que apresentam mais de um significado nos múltiplos contextos, e isso as faz terem interpretações ambíguas ou mesmo serem consideradas polissêmicas. A partir das várias acepções de uma palavra polissêmica, pode ocorrer que um significado básico dê origem a significados derivados - é o caso da palavra classificação.

Este estudo surgiu com base na leitura do artigo "Classification", de autoria de Birger Hjørland, publicado em 2017, no qual o autor suscita tal questão. Assim, estudar esta perspectiva interdisciplinar do significado da palavra classificação, a partir de sua definição, tem o intuito de traçar o seu contexto histórico e a sua evolução na área de Ciência da Informação e Biblioteconomia.

Neste artigo, o foco, portanto, é a palavra classificação, que tanto pode ser considerada uma disciplina básica no campo da Biblioteconomia, como também um conceito importante em outros campos do conhecimento.

A classificação é objeto central de pesquisas em muitas ciências. Nos estágios iniciais, os estudos sobre ela partiram dos estudos filosóficos; mais tarde, tiveram uma abordagem educacional e psicológica; e, durante a década de 1940, começaram a ficar mais evidentes os estudos sobre a Taxonomia biológica, e, na Matemática, com a intenção de agrupamento. Da mesma forma, Garcia Marco e Esteban Navarro (1993) dão destaque para áreas como: Matemática, Psicologia, Filosofia, Linguística e Ciência da Computação, que têm estudos sobre a classificação. Além dessas áreas, outros campos também estão 
envolvidos, de uma forma ou de outra, em estudos sobre esse termo, como a Antropologia (por exemplo, sistemas parentais, etnolinguísticas) e também a Biologia (Botânica e Zoologia (Linnaeus)). Garcia Marco e Esteban Navarro (1993) acrescentam que:

na verdade, todas as ciências estão preocupadas com o problema ou classificação; as ciências sociais e humanas porque eles estão lidando com processos humanos dos quais a classificação é um aspecto central; todos os outros porque classificação é uma parte crucial da metodologia científica (GARCIA MARCO; ESTEBAN NAVARRO, 1993, p. 127).

Hjørland (2017, p. 122) cita o trabalho de McKenna; Bell (1998, 1133), no qual os autores fornecem uma visão geral da história e da teoria da classificação e afirmam: "A palavra 'classificação' não fazia parte da literatura científica até as últimas décadas do século XVIII. O primeiro uso de que temos conhecimento ocorre em um artigo botânico do Marquês de Condorcet (1777: 35)".

É sabido que os estudos sobre classificação em geral e sobre a classificação bibliográfica estão em constante evolução. A história da classificação do conhecimento teve como partida o modelo estabelecido por Aristóteles, que prevaleceu durante aproximadamente dois mil anos (300 aC a 1600) e começou a decair no final da Idade Média. Bowker e Star (1999, p. 1) observam que "classificar é humano", pois o ser humano classifica objetos quase por instinto e inconscientemente. E acrescenta que, apesar de a classificação ser considerada uma atividade

antiga, pode ser considerada, também, uma atividade moderna, pois continua sendo necessária com o uso de estruturas mais dinâmicas, haja vista sua importante contribuição, principalmente com a sua característica multidimensional, que facilita a representação de informações de assuntos interdisciplinares e multidisciplinares em bases de dados e na Web.

A classificação em geral e a classificação bibliográfica, em particular, foram definidas de maneiras diversas por diferentes autores e estudiosos. Dependendo da perspectiva, é vista como uma ciência, isto é, relacionada a um campo de estudo e suas atividades; como o produto que resulta do processo de divisão e, simultaneamente, é a ferramenta utilizada para realizar o processo de classificação. No entanto, pode-se ver que as definições são orientadas para um propósito específico. Em todas as abordagens, mantém-se um ponto em comum: estabelecer uma ordem ou organização das coisas e dos pensamentos.

Assim sendo, pretende-se, neste estudo, lançar um olhar na literatura específica para mapear as definições de "classificação", a partir de autores seminais da área, com o objetivo de organizar e sistematizar 
as ideias principais representadas nessas definições para evidenciar a evolução e o contexto histórico desse termo.

\section{Classificação}

\subsection{Um olhar a partir da definição}

Classificação é um conceito fundamental em várias áreas, visto que é um processo de nomear e ordenar um universo do conhecimento. Por isso, quando se refere à história da dassificação, sempre se remonta ao início da humanidade, uma vez que é característica inerente do homem a capacidade de distinguir objetos por meio de suas semelhanças e diferenças, que são distintas em suas identidades para os seres humanos.

No início, porém, essa sistematização do conhecimento era realizada de forma intuitiva, e não de forma esquemática, como foi realizada a partir do século XVIII, e, conforme Dahlberg (1979, p. 324), "a arte de elaborar os esquemas não era chamada de 'classificação' até quase 200 anos depois, no final do século XVIII". No entanto, Fosket (1980, p. 2) constata que "a história da classificação do conhecimento mostra que esquemas de ordenação de conhecimentos ou documentos que contenham conhecimento sempre, e inevitavelmente, refletem as filosofias e teorias do conhecimento".

De acordo com Körner (1976, p. 3), para compreender o mundo, os homens empregam constantemente três métodos de organização, que permeiam todo o pensamento: (1) a experiência em diferenciar um objeto específico e seus atributos, como, por exemplo, quando se distingue entre uma árvore e seu tamanho, ou suas relações espaciais com outros objetos; (2) a distinção entre objetos no todo e as partes que os compõem, por exemplo, quando se compara uma árvore com as partes que a compõem; (3) a formação e a distinção entre diferentes classes de objetos, por exemplo, quando se forma uma classe de todas as árvores e uma outra com todos os tipos de pedras; e quando se faz a distinção entre elas. A partir dessas colocações, pode-se inferir que a classificação está no cotidiano de todos os comportamentos humanos, mesmo nas atividades mais elementares. É um processo, quase que instantâneo, que dá habilidade ao ser humano de analisar, sintetizar, ordenar seu intelecto e estruturar seu conhecimento cognitivamente.

Isso posto, pode-se dizer que uma das características principais da classificação é a capacidade de sistematizar os conceitos a partir de suas semelhanças e diferenças, de forma dicotômica. De acordo com Dalhberg (1978, p. 104), "o conceito é a unidade de conhecimento que surge pela síntese dos predicados necessários relacionados com determinado objeto e que, por meio de sinais linguísticos, pode ser comunicado".

O objetivo da definição de um conceito é a especificação de sua finalidade e, nessa especificidade, rotular uma ou mais variáveis do 
universo; ou seja, o conceito é formado por meio de predicados relacionados com o objeto do mesmo conceito, sendo que cada predicado fornece uma característica. No caso da classificação, o conceito deve ser definido para ser inserido dentro do contexto de uma área, a partir de critérios e princípios a serem observados para agrupá-los em classes e subclasses no esquema de classificação. Segundo Piedade (1983), a palavra classificação vem do latim classis, que designava os grupos em que dividia o povo romano com base no nascimento e riqueza, sendo cunhada por Zedler, em 1733, no Universal Lexicon.

A classificação, por ser uma palavra que perpassa várias áreas do conhecimento, conforme referido anteriormente, pode ter definições diferenciadas, dependendo da abordagem, do contexto semântico e da área do conhecimento em que seu conceito se expressa. Entretanto, a classificação, em essência, significa dividir em clusters, agrupar, classificar, organizar, ordenar, classificar e relacionar uma entidade com as outras. Assim, ela é um processo mental por meio do qual agrupamos ou distinguimos coisas com base em características gerais.

Dependendo do ponto de vista, a classificação é considerada uma disciplina, mas também pode ser o produto que resulta do ato de classificar e, simultaneamente, é a ferramenta utilizada para realizar o processo de classificação. Da mesma forma, classificar é o processo que dá origem a um sistema estruturado, e classificação é o dispositivo pelo qual a construção é tomada geralmente, mas não apenas, a priori.

Em 1929, Henry E. Bliss já apontava esta questão em relação à definição da palavra classificação e escreveu que:

o termo, como outros derivados do inglês que terminam em íon, é ambiguamente usado tanto no sentido predicativo quanto no substantivo, para uma ação ou para um ato, às vezes refere-se ao processo e às vezes ao produto (BLISS, 1929, p. 142).

Para solucionar esse problema de ambiguidade, o autor sugeriu três definições derivadas da palavra "classe" como um substantivo:

(1) o verbo "to class" denota associar, referir, ou atribuir algo a alguma classe, ou várias coisas às suas respectivas classes, conforme possa ser necessário ou relevante para o interesse envolvido. Este verbo é usado não apenas transitivamente, mas às vezes intransitivamente. Assim, pode-se dizer que o azeite é considerado um luxo; (2) o verbo "classify" significa principalmente fazer ou conceber uma classe, ou classes, a partir de uma pluralidade de coisas, e secundário para organizar classes em alguma ordem ou relacioná-las em algum sistema de acordo com algum princípio ou concepção, propósito ou interesse [...]. Esses 
três processos, classificar, formar classes e organizar classes, estão tão implicados que não é fácil separá-los em pensamento ou terminologia; no entanto, propomos aqui que isso deve ser feito como conducente à precisão neste estudo. Há uma distinção importante entre atribuir uma coisa ou coisas a alguma classe ou classes e organizar as classes em alguma ordem ou sistema. [...]; (3) uma "classification" é uma série ou sistema de classes arranjadas em alguma ordem de acordo com alguns princípios ou concepção, propósito ou interesse, ou alguma combinação de tais (BLISS, 1929, p. 142-143).

Essa definição é citada em Hjørland (2017), que a considera uma das mais elucidativas para a palavra classificação.

Posteriormente, Sayers, em 1950, também sugere que essa palavra tenha quatro significados, e faz as seguintes distinções entre eles:

(a) o processo mental pelo qual nossos conceitos mentais... são reconhecidos por ter semelhanças ou unidade, e por essa relação ou unidade são estabelecidas as relações uns com os outros. Esse é um significado lógico e real; (b) o ato de organizar coisas reais... de modo que representem o arranjo abstrato. Isso é uma classificação prática; (c) uma tabela escrita ou impressa de termos que representam um sistema de classificação. Isso é chamado de esquema de classificação; (d) o ato de colocar as coisas ou livros em seus lugares apropriados a partir do uso de um esquema de classificação. Isso é classificar (SAYERS, 1950, p. 79).

Outro autor que teve a mesma preocupação em elucidar as diferentes abordagens que a classificação pode abranger foi Shera (1965, p. $120 ; 127)$, em seu livro Libraries and the Organization of Knowledge, considerando que

"(1) Classificação é a cristalização ou formalização do pensamento inferencial, nascido da percepção sensorial, condicionada pela operação do cérebro humano e moldada pela experiência humana. Ela está na base de todo pensamento, mas é pragmática e instrumental; [...] (2) classificação bibliográfica é uma lista de termos que são especificamente diferentes uns dos outros, usados para descrever o conteúdo do assunto dos registros gráficos, incluindo to do conhecimento definido pelos limites do esquema, infinitamente hospitaleiro no que diz respeito às diferenças significativas entre o conceito, com um arranjo que é linear, único e significativo para o usuário, e que quando aplicado a registros gráficos, resulta no arranjo dos próprios registros; [...] (3) Ele [0 bibliotecário] deve apreciar a classificação, não como uma 
ferramenta, mas como uma disciplina que busca alcançar uma melhor compreensão dos padrões de mudança de pensamento e os pontos de contato nos quais eles podem ser relacionados a unidades específicas de informações registradas.

Por sua vez, S. R. Ranganathan, em seu Prolegomena to Library Classification (1967), no capítulo CP, entre as páginas 77 e 78, discute exaustivamente o significado da palavra classificação. O autor considera este termo polissêmico e, por isso, propõe esclarecer as acepções que essa palavra pode ter para evitar ambiguidade em seu uso. Para ele, no senso comum, a classificação pode ter duas abordagens: uma enquanto a divisão que implica a classificação de objetos em dois ou mais clusters; e, adicionalmente, outra, que denota a disposição desses clusters em uma sequência predeterminada. No caso da classificação bibliográfica, a sequência de objetos, ou seja, de documentos, é, portanto, sistematizada por meio da exploração da notação que é refletida no momento em que o documento é catalogado ou emprestado. Ranganathan $(1967,1973 b)$ vê a classificação como uma forma de descobrir o conteúdo expresso em uma unidade escrita ou expressão do pensamento. Para o autor, as ideias são analisadas, agrupadas e lançadas numa sequência útil - isso é classificação - não só para facilitar a memória, mas também para ajudar a pensar. Em todas essas etapas, a classificação pode ser considerada inexorável.

Assim, com o intuito de elucidar esta questão de homonímia que a palavra classificação pode alcançar, Ranganathan examinou várias abordagens em que ela foi usada, e trouxe à tona cinco definições com perspectivas diferentes. A primeira ele chamou de significado 1, no sentido mais primitivo do processo de classificar, que ocorre no dia a dia do ser humano, a partir de uma divisão dicotômica do universo, representando as coisas e ideias em grupos, sem notações. No significado 2, Ranganathan esclarece que, nessa perspectiva, a classificação é vista como um processo inerente a toda pessoa. Para o autor, nesse significado está a necessidade de expressar, de falar, ou seja, uma necessidade neural de processar a informação. Nesse caso, segundo esse autor, o trabalho dos filósofos e o dos taxonomistas estariam inseridos nesse significado. Encontra-se nele o processo de agrupamento, no qual ocorre o processo de divisão do universo em grupos e o arranjamento em classes, podendo ser divididas infinitamente, e representadas por notações. Já quanto ao significado 3, considera-se o mesmo processo realizado no significado 2, acrescentada a representação de cada entidade por meio de um número ordinal retirado de um sistema hierárquico, para garantir a sequência estabelecida. O processo de classificar nesse significado exige um alto grau de abstração. 
A classificação, no significado 4, é a do significado 3, quando ocorre o agrupamento completo de todo universo, e dele surgem entidades e pseudoentidades que vão sendo organizadas em uma sequência lógica e filiatória. Já a classificação no significado 5 é a classificação no significado 4 com todas as entidades e pseudoentidades, que não pertencem ao universo do qual foram removidas, e mantendo somente as que realmente fazem parte do sistema no todo.

Ranganathan salienta que a classificação no significado 4 não é muito utilizada, sendo a classificação nos significados 3 e 5 as mais demandadas. O significado da classificação 5 é usado quando o universo classificado é infinito, e existem poucas entidades desconhecidas. Nesse caso, o autor sugere que a classificação no significado 5 é a mais praticada na profissão de bibliotecário, pois o objetivo principal das bibliotecas é sistematizar e organizar os documentos, e a classificação na biblioteca pressupõe o uso da notação de assunto. Deve-se lembrar que nesse significado 5 da classificação, a entidade individual não aparece em todo agrupamento, sendo substituída pelas classes, e cada classe, incluindo a classe geral, forma outras classes; diferentemente da classificação no significado 2, que tem apenas um esquema para as classes associadas a ele. Para Ranganathan, o significado do termo classificação deve ser considerado do ponto de vista da abordagem 5 .

Essa explanação apresentada por Ranganathan é considerada por alguns como sendo definições bem precisas, bastante explícitas e que atendem ao melhor uso para esse propósito. No entanto, ressalta-se aqui a dificuldade de expressar-se o pensamento de Ranganathan, pela sua forma peculiar de expressão, além da terminologia bem específica em seus escritos.

Nos mesmos moldes das definições supracitadas, quatro décadas depois, Ingetraut Dahlberg (2010), considerando, também, os vários significados que a palavra classificação pode ter, apresenta quatro acepções, da seguinte forma:

(1) classificação no sentido de sistema de classificação, isto é, um sistema de classes organizadas em ordem hierárquica ou facetada; (2) classificação no sentido de classificar, isto é, estabelecer um sistema de classes; (3) classificação no sentido de classificar, isto é, relacionar as classes de um sistema de classificação a objetos ou sujeitos da realidade; e (4) classificação no sentido de ciência da classificação, isto é, relacionada a este campo de estudo e suas atividades (lista tipográfica adicionada) (DAHLBERG, 2010, p. 2941).

Em suma, infere-se que, no âmbito da Biblioteconomia e Ciência da Informação, mais especificamente, o termo classificação é usado para se 
referir a três conceitos distintos, mas relacionados: (1) um sistema de classes, ordenado de acordo com um conjunto predeterminado de princípios e usado para organizar um conjunto de entidades; (2) um grupo ou classe em um sistema de classificação; e (3) o processo de atribuição de entidades a classes em um sistema de classificação.

Optou-se por apresentar detalhadamente essas definições propostas por estes quatro autores, seguindo uma ordem cronológica, por considerar que elas irão balizar a análise de conteúdo que será realizada na amostra deste estudo. Essa amostra se encontra em Hjørland (2017, p. 122), que será apresentada posteriormente.

\subsection{A Teoria}

O arcabouço teórico do processo classificatório focaliza-se nas classificações filosóficas com os estudos de Platão. Posteriormente, Aristóteles contribuiu com dois alicerces para o processo classificatório: 1) a divisão dicotômica das coisas, em hierarquias de gênero e espécie, com a aplicação de princípios classificatórios, agrupando os semelhantes e separando os diferentes; 2) desenvolvimento de cinco predicados (relações) existentes em um arranjo lógico: gênero (classe), espécie (tipo), diferença (característica específica), propriedade (atributo) e acidente (qualidade).

Outra contribuição para a teoria da classificação vem de Porfírio, filósofo do século IV, que aplicou o princípio de Platão e Aristóteles para criar seu processo denominado "Árvore de Porfírio". Divergente de Aristóteles, Porfírio não tratou o conceito de substância apenas como gênero/espécie, mas como uma classificação lógica da substância, sistematizando a noção de ideias gerais, chegando às mais específicas, usando os predicados de Aristóteles.

Também Bacon, em 1605, contribuiu com fundamentos para o processo classificatório, quando propôs a divisão das ciências segundo a lógica das faculdades humanas: História (memória), Poesia (imaginação) e Filosofia (razão), dando ênfase aos assuntos tratados nos documentos e não mais às coleções em si. Adicionalmente, Bacon publicou uma trilogia de livros sobre teoria da classificação, demonstrando o primeiro método científico indutivo. Esse autor recomenda o uso de três tábuas para abordagem: 1) a tábua de presença: registro da presença das formas que se investigam; 2) a tábua de ausência (ou declinação): controle de situações nas quais as formas pesquisadas se revelam ausentes; 3 ) a tábua de comparação (ou graduações): registro das variações em que as referidas formas se manifestam.

No contexto da Biblioteconomia, as teorias de classificação na realidade muitas vezes significam "ideias" ou "princípios" de classificação (SMIRAGLIA, 2002). A teoria da classificação bibliográfica teve seu 
desenvolvimento com E. D. Richardson (1930) com o livro Classification: theoretical and practical, publicado pela primeira vez em 1901. Em seus estudos, o autor reconheceu a necessidade de desenvolver princípios de classificação para que, a partir de seus embasamentos, pudesse realizar a modificação e a revisão dos esquemas já existentes - para classificação bem como para a avaliação dos esquemas de classificação. Além disso, constatou a necessidade do desenvolvimento de uma teoria geral para classificação bibliográfica. O objetivo de Richardson (1930) em desenvolver uma teoria geral de classificação era tanto contribuir para a compreensão teórica dos princípios classificatórios em biblioteconomia quanto estabelecer princípios que podem guiar a construção prática de classificações bibliográficas (DOUSA, 2010, p. 17).

Posteriormente, W. C. Bliss (1929) trouxe um contexto filosófico na abordagem sistemática no desenvolvimento da teoria da classificação bibliográfica, indicando a forma como essa classificação deve refletir a abordagem do especialista. Ele trouxe uma espécie de consenso educacional na maneira de utilizar os sistemas de classificação. Outra contribuição para a teoria da classificação bibliográfica veio de $\mathrm{E}$. Wyndham Hulme (1950), quando considerou a garantia literária como a mais importante base para a realização da classificação de um documento.

No entanto, foi Shiyali Ramamrita Ranganathan (1892-1972), um dos estudiosos que mais contribuiu para a teoria da biblioteconomia no século $X X$, com seus estudos sobre a teoria da classificação facetada, e a idealização de sua Classificação de Dois Pontos, também conhecida como Classificação em Facetas ou Classificação Analítico-Sintética. Um sistema facetado reconhece muitos aspectos em um único conceito ou classe geral e tenta sintetizar esses aspectos, de maneira a melhor descrevê-los. A facetação mostrou que a relação entre assuntos, feita por meio do símbolo de dois pontos (:), pode ser infinita, explicitando que o conhecimento pode ser multidimensional e que suas relações podem tomar rumos diferentes, dependendo de como a síntese entre vários conceitos múltiplos seja realizada (VICKERY, 1980). Ranganathan criou 46 cânones para a análise de facetas, 13 postulados e 22 princípios para aplicar a análise facetada aos sistemas de classificação.

Nota-se, portanto, que todas as bases teóricas do processo de classificação, a partir de seus princípios e cânones, buscam promover uma classificação sistemática, lógica, que reflete crítica e filosoficamente sobre os elementos de ligação que servem para indicar a reunião e os relacionamentos entre seus conceitos. 


\subsection{O Processo}

O processo de classificar envolve a atribuição ordenada e sistemática de cada entidade a apenas uma classe dentro de um sistema de classes mutuamente exclusivas e não sobrepostas, com base em semelhanças e diferenças. Bliss (1929, p. 143) considera que o verbo classificar tem dois significados:

(1) fazer ou conceber uma classe ou classes, a partir de uma pluralidade de coisas. Implica a semelhança de coisas para formar o núcleo de uma classe, e que a partir daí outras coisas semelhantes são referidas ou atribuídas à classe; (2) organizar as classes em alguma ordem ou relacioná-las em algum sistema de acordo com algum princípio ou concepção, propósito ou interesse. Implica não apenas que as coisas são classes e que as classes são formadas ou concebidas, mas também que as próprias classes são organizadas e sistematizadas (BLISS, 1929, p. 143).

Para Vickery (1980, p. 23), "classificar, na acepção mais simples do termo, é reunir coisas e ideias que sejam semelhantes entre si, e separar as que apresentam diferenças". Assim, o processo classificatório se baseia no estabelecimento de classes de elementos que tenham pelo menos uma característica comum, podendo esses elementos serem organizados segundo um ponto de vista, ou a finalidade da classificação.

É considerado um processo sistemático realizado de acordo com um conjunto de princípios estabelecidos, que orientam a estrutura e o relacionamento das classes. Assim, a classificação como um processo consiste essencialmente em reconhecer as formas lógicas orientadas para o propósito das entidades de um universo reconhecer grupos e suas relações mútuas. Do mesmo modo, é considerado um processo de colocar livros e outros materiais em uma sequência lógica na estante, para ajudar os usuários no acesso aos documentos; requer um estudo aprofundado sobre a prática na técnica de classificação de livros, conhecimento dos detalhes e manuseio do esquema de classificação. De acordo com Shera e Egan (1956), o verbo classificar tem dois significados: (1) a concepção das classes a partir da pluralidade das coisas, e (2) o arranjo das classes de acordo com uma ordem conforme determinados princípios.

Enquanto para Ranganathan (1973a, p. 21; p. 25) "classificar, no sentido primitivo, é dividir o universo do discurso - concreto ou conceitual, coisas ou ideias - em dois grupos [...] colocar as coisas em alguma ordem mais ou menos útil, ou mencionar ideias em alguma ordem mais ou menos útil - parece ser comum com o homem". Ademais, Ranganathan (1973b) considera classificar um processo de divisão das entidades de um universo, organizando os grupos em uma sequência definida que pode organizar documentos por qualquer princípio (autor, 
título, data, idioma, assunto, tamanho, etc.), mas o princípio mais popular, útil e difícil é o assunto. $O$ autor ressalta que, se puder recuperar informações por assunto, você pode recuperar por qualquer critério.

Assim sendo, pode-se dizer que o processo de classificar pode ser aplicado de duas maneiras distintas e complementares na atividade de organização: a) permite organizar o conhecimento em si, por meio de sua representação numa estrutura taxonômica que enumera seus ramos principais e subsidiários; e b) possibilita a organização de documentos, mantendo-os agrupados conforme o assunto de que tratam (BATLEY, 2005). Dessa forma, na perspectiva da Biblioteconomia e Ciência da Informação (BCI), o ato de classificar significa ordenar e dispor objetos (ou ideias) em classes a partir de determinado critério.

\subsection{O Sistema}

Os sistemas de classificações bibliográficas possuem caráter normativo e são utilizados para organizar acervos de modo a facilitar a recuperação da informação. Langridge (1977, p. 38) os define como "um mapa completo de qualquer área do conhecimento mostrando todos os seus conceitos e suas relações". Como já mencionado anteriormente, esses sistemas são concebidos considerando-se o universo como um todo que se divide em classes e subclasses de acordo com certas características comuns. Segue uma progressão do geral ao específico, formando uma estrutura hierárquica na qual os assuntos que compõem cada nível são organizados de acordo com sua afinidade ou possíveis relações. Svenonious (1992) explica a classificação como uma ciência para elaborar sistemas de classificação, afirmando que

[...] é uma ciência produtiva na medida em que seu objetivo é produzir sistemas de classificação. Mas como esses sistemas são orientados para a ação, sendo esta ação organizadora do universo dos assuntos, a disciplina pode ser classificada entre as ciências práticas. E então, uma vez que a disciplina busca demonstrar verdades gerais sobre seus objetos de estudo, a saber, o universo de assuntos e sistemas de classificação, ele participa das ciências teóricas (SVENONIOUS, 1992, p. 176-177).

Os esquemas de classificação ajudam um classificador a representar o conteúdo do assunto de cada documento por notações apropriadas. Reitz (2004) aponta os seguintes objetivos de um esquema de classificação:

(1) Para fornecer um endereço na prateleira - para atribuir um local específico para cada documento nas prateleiras da biblioteca; (2) Para agrupar itens - colocar documentos sobre o mesmo assunto ou 
assuntos semelhantes juntos nas prateleiras da biblioteca para facilitar os usuários a encontrar itens sobre o mesmo assunto em um lugar dentro da biblioteca; (3) Para vincular itens - para permitir que os usuários procurem itens por meio de um catálogo de biblioteca, use números de telefone como referências para localizar itens nas prateleiras; a classificação é usada como um link entre o registro do catálogo de um item e o próprio item na prateleira; (4) Para habilitar a navegação - usando a estrutura de uma classificação bibliográfica para navegar uma coleção nas estantes da biblioteca ou em uma coleção eletrônica (REIZ, 2004, p. 224).

Os sistemas de classificação bibliográfica são sistemas verbaissimbólicos, geralmente constituídos por três elementos: 1) um esquema de classificação, agrupando elementos sistematicamente, por suas semelhanças; 2) uma notação da classificação (número de chamada); 3) um índice alfabético, que facilita a busca do usuário. Neles, a divisão por classes baseia-se em dois princípios básicos: a) de divisão, que respeita critérios preestabelecidos, obedecendo a uma característica específica de divisão; b) de classes simples e classes compostas, nas quais a classe simples identifica uma única característica, em que não cabem mais subdivisões, e a classe composta identifica mais de uma característica, em que podem caber mais subdivisões.

\subsection{Classificação nas disciplinas}

O estudo sobre a classificação pode assumir diferentes contextos de acordo com a área do conhecimento em que ela se insere, caracterizando seu caráter interdisciplinar. Satija (1998) indica seis disciplinas com as quais a classificação pode estar relacionada: (1) Lógica - classificação usa métodos da lógica; (2) Psicologia - todo aprendizado e memória envolve classificação, pois a mente funciona por classificação; (3) Filosofia - a classificação é inerente a qualquer definição. Toda teoria é classificação, uma vez que "identifica possíveis relações entre variáveis-chave e sugere como e por que eles estão relacionados" (SOPER et aI., 1990); (4) Epistemologia - é a teoria da natureza e organização de conhecimento. A relação entre classificação e epistemologia é intrínseca; (5) Linguística Classificação é nomear. Classificação é uma linguagem de indexação. Os conceitos existem na linguagem. Linguística, terminologia e semântica são fundamentais à classificação. A linguagem é um instrumento na organização do conhecimento; (6) Biblioteconomia e Ciência da Informação - Esquemas de classificação formam os fundamentos de qualquer tipo de vocabulário estruturado e controlado; representam os assuntos indexados; a elaboração de índices de assuntos alfabéticos para catálogos classificados é derivada de esquemas de classificação em uso.

Alguns estudos apontam que a classificação, enquanto área, cumpre todos os requisitos para tornar-se uma disciplina acadêmica independente, 
pois segue diferentes escolas de pensamento, sua prática é baseada em uma teoria sólida, ela se ampara em diversas escolas teóricas, possui um corpo literário coerente em todas as formas, sendo produzida incessantemente. Existe uma diversidade de livros didáticos, anuais, relatórios de pesquisa e artigos de periódicos, inclusive revistas dedicadas à área. Conferências regional, nacional e internacional sobre classificação também são realizadas para compartilhar novos conhecimentos e apresentar pesquisas, bem como sociedades nacionais e internacionais exclusivamente dedicadas à promoção da classificação de estudos e pesquisas. Além disso, os estudos sobre classificação são ministrados em cursos de Biblioteconomia e Ciência da Informação em nível universitário, formando sempre novos profissionais e pesquisadores (SATIJA, 1998, p. 34).

Procurou-se, nesta seção, abordar a classificação a partir de sua teoria, as definições, o processo, enquanto sistemas de classificação e as disciplinas que se relacionam com esse termo. Nas próximas seções, apresenta-se, primeiramente, a metodologia, seguida da análise da amostra escolhida, com o uso do método da análise de conteúdo (BARDIN, 2011); depois das reflexões, discorre-se sobre os resultados e parte-se para as considerações finais.

\section{Metodologia}

Esta pesquisa, como um todo, tem uma abordagem qualiquantitativa, pois, no seu decorrer, será usada a análise dos dados tanto qualitativa quanto quantitativamente, utilizando o método análise de conteúdo. Caracteriza-se como uma pesquisa exploratória e descritiva, pois parte-se de um levantamento bibliográfico, buscando analisar as abordagens e características nas quais a palavra classificação aparece na literatura a partir do contexto filosófico e bibliográfico. Para isso, propôsse, primeiramente, elaborar uma revisão narrativa da literatura, para: (1) mapeá-la sobre o contexto da palavra classificação e (2) traçar o contexto histórico e a evolução da classificação a partir do significado do termo em uma perspectiva interdisciplinar.

Nesse contexto, foram feitos os seguintes questionamentos para direcionar este estudo: (1) quais são as abordagens em que o termo classificação aparece na literatura? (2) qual a evolução histórica teórica das definições de classificação? (3) quais são as áreas de conhecimento que subsidiam as diversas acepções sobre classificação?

\subsection{Amostra}

A amostra desta pesquisa são as 40 definições sobre classificação de diversos autores, apresentadas em ordem cronológica, de c.370 BC a 2016, selecionadas a partir do Apêndice do artigo "Classification", de 
autoria de Birger Hjørland, publicado em 2017, no periódico Knowledge Organization e apresentadas, também, como Anexo 1 neste artigo. O artigo é publicado em língua inglesa, portanto, reflete as definições publicadas nesse idioma, e pretende, por meio de uma avaliação dessas definições, fazer um panorama histórico conceitual da classificação a partir das diversas manifestações do termo. Ressalta-se que, apesar desta autora considerar que existem outras definições importantes na literatura, preferiu-se não ampliar a amostra já compilada por Hjørland, para evitar outras variáveis.

\subsection{O método: a Análise de Conteúdo}

Qualquer técnica de análise de dados, em última instância, significa uma metodologia de interpretação. Como tal, possui procedimentos peculiares, envolvendo a preparação dos dados para a análise, visto que esse processo "consiste em extrair sentido dos dados de texto e imagem" (CRESWELL, 2007, p. 194). Entre as técnicas com abordagem sistemática e qualitativa no contexto de investigação empírica realizada nas ciências humanas e sociais, encontra-se o método de Análise de Conteúdo (AC).

Para Bardin (1977), a análise de conteúdo designa:

um conjunto de técnicas de análise das comunicações visando a obter, por procedimentos sistemáticos e objetivos de descrição do conteúdo das mensagens, indicadores (quantitativos ou não) que permitam a inferência de conhecimentos relativos às condições de produção/recepção (variáveis inferidas) destas mensagens (BARDIN, 2011, p. 48).

Uma característica importante da análise de conteúdo é sua capacidade de produzir inferências, como uma importante característica, que dá ao método relevância teórica a partir de comparações de informações descritivas. Bardin (1977) enfatiza que "a intenção da análise de conteúdo é a inferência (ou conhecimentos relativos às condições de produção - ou eventualmente, de recepção); inferência esta que recorre a indicadores (quantitativos ou não) " (BARDIN, 1977, p. 38).

Apesar de possuir qualidades tais como reduzir a complexidade da informação analisada em descrições das características das unidades e facilitar as comparações entre essas unidades, umas das suas maiores limitações é a abordagem metodológica quantitativa, que pode dificultar a real interpretação dos conteúdos.

Existe na literatura, em relação às etapas inerentes à análise de conteúdo, diversas propostas com uso de terminologia diferentes, porém todas têm em comum o processo de análise e sistematização da informação. Neste artigo, optou-se por seguir as etapas sugeridas por Bardin (2011), por serem as mais citadas na literatura da área. Esse 
método é composto de três fases básicas:(1) pré-análise, (2) exploração do material e (3) tratamento dos resultados, inferência e interpretação e atividades.

A primeira etapa, pré-análise, é conhecida como um período que tem o objetivo de organizar e sistematizar as ideias iniciais para conduzir os procedimentos para análise. Primeiramente, escolhe-se um conjunto de dados para serem submetidos à análise, que ocorrerá por meio da sistematização dos assuntos a partir de cinco fases: (a) leitura flutuante, para conhecimento inicial do texto; (b) escolha dos documentos, que é a determinação do corpus a ser analisado; (c) determinação dos pressupostos e objetivos; (d) elaboração de indicadores e (e) preparação do material.

$\mathrm{Na}$ segunda etapa, ocorre a exploração do material a partir da operação de codificação e decomposição das publicações selecionadas, e a classificação e a criação de sistemas de codificação. Nesse processo, determinam-se ou se identificam as unidades de registro (UR), que serão as unidades base para a contagem da frequência, e as unidades de contexto (UC), que têm o papel de significação que irá permitir a compreensão de uma UR. Essa é uma fase fundamental no método AC, pois possibilitará, ou não, melhores interpretações e inferências, dependendo da maneira como for realizada. Duas técnicas podem ser utilizadas nesta fase: (1) análise léxica para avaliar as palavras portadoras de sentido relacionadas ao objeto de pesquisa, para reconhecer a terminologia mais utilizada pela comunidade específica da área, e (2) análise categorial, a qual deve obedecer aos critérios de escolha e de delimitação que orientam a dimensão do estudo sobre os temas relacionados ao objeto.

Já a terceira etapa consiste no tratamento, na inferência e na interpretação dos resultados, quando se faz um resumo e se destacam as informações para análise, elementos que possibilitam interpretações inferenciais. Os resultados das análises quantitativas ou qualitativas são descritos e representados por meio de gráficos ou tabelas. Esse foi o método adotado para este estudo, e serão apresentados a seguir sua aplicação e os resultados.

\section{Aplicação da análise de conteú do na amostragem}

A amostragem consiste na apresentação de 40 definições, em inglês, sobre classificação, de diversos autores, em ordem cronológica, publicadas como apêndice do artigo "Classification", de autoria de Birger Hjørland, da revista Knowledge Organization, v. 42, n. 2, $2017 . \quad$ Para analisar essas definições, aplicou-se a técnica da análise de conteúdo em três etapas. 


\section{Primeira etapa}

Na pré-análise, decidiu-se pela escolha do artigo "Classification", de autoria de Birger Hjørland, publicado em 2017, sendo considerado um artigo que atendia aos pré-requisitos predefinidos. Realizou-se a leitura flutuante, para conhecimento do texto e da formulação de pressupostos. Como afirma Bardin (2009, p. 121), "a escolha dos documentos depende dos objetivos, ou, inversamente, o objetivo só é possível em função dos documentos disponíveis". Assim, após análise do conteúdo do artigo, fezse o recorte, selecionando as 40 definições contidas no apêndice, levando em consideração que o próprio documento já trazia insumos suficientes para alcançar o objetivo proposto. Em função de ocupar muitas páginas, neste artigo se torna inviável apresentar todo o Apêndice com as definições na íntegra.

Após uma leitura flutuante das definições sobre classificação, formularam-se alguns pressupostos: (1) a palavra classificação possui significados em múltiplos contextos por ter um caráter interdisciplinar; (2)O arcabouço teórico do processo classificatório teve sua base nas classificações filosóficas com os estudos de Platão e, posteriormente, com Aristóteles;(3) a Biblioteconomia e Ciência da Informação, a classificação pode ser vista como ciência, como um produto resultado do processo de divisão, e como a ferramenta utilizada para realizar o processo de classificação.

\section{Segunda etapa}

Caracterizou-se pela exploração do material para criação de um conjunto de categorias que são classificadas e codificadas, com a identificação das Unidades de Registro (UR), que são os conceitos relevantes no texto; e as Unidades de Contexto (UC), que são as categorias mais gerais que contextualizam e dão significado às URs. Para isso, fez-se a exploração do material em dois estágios: primeiramente, (1) análise léxica para avaliar as palavras portadoras de sentido nas definições, para reconhecer a terminologia mais utilizada pela comunidade específica da área e, posteriormente, (2) a análise categorial.

No primeiro estágio, realizou-se uma análise automática das 40 definições, utilizando o software Jupyter ${ }^{1}$ para (1) mapear a terminologia mais utilizada pelos autores das definições sobre classificação, (2) verificar o número de ocorrência desses vocábulos no texto, e (3) mensurar quais palavras aparecem no mesmo contexto. Para fazer a contagem dos termos portadores de sentidos de maior ocorrência,

\footnotetext{
${ }^{1}$ https://jupyter.org/
} 
determinou-se que os termos classes/class, things/thing, objects/object, classifications/classification, characters/characteristics fossem contados juntos para resolver a questão dos termos utilizados no singular e plural; e selecionaram-se como palavras vazias, as stopwords "may, be, can, according, to, which, thought, one, also, chap, see, general, number, problem, together, called, true". Como resultado, optou-se por apresentar somente os 50 primeiros termos que obtiveram até a frequência 5, dos 91 extraídos da análise léxica, por motivo de espaço no texto, conforme Tabela 1.

Tabela 1 - Resultado da análise léxica das 40 definições sobre a palavra classificação

\begin{tabular}{|c|c|c|c|c|c|}
\hline $\begin{array}{c}\text { Posição/orde } \\
\text { m }\end{array}$ & Termo & $\begin{array}{c}\text { Frequênc } \\
\text { ia }\end{array}$ & $\begin{array}{c}\text { Posição/orde } \\
\text { m }\end{array}$ & Termo & $\begin{array}{c}\text { Frequênc } \\
\text { ia }\end{array}$ \\
\hline 1 & $\begin{array}{l}\text { classificatio } \\
\mathrm{n}\end{array}$ & 120 & 26 & sequence & 6 \\
\hline 2 & class & 53 & 27 & categories & 6 \\
\hline 3 & thing & 24 & 28 & level & 6 \\
\hline 4 & sense & 24 & 29 & $\begin{array}{l}\text { categorizati } \\
\text { on }\end{array}$ & 6 \\
\hline 5 & object & 21 & 30 & natural & 5 \\
\hline 6 & $\begin{array}{l}\text { characteristi } \\
\text { cs }\end{array}$ & 14 & 31 & parts & 5 \\
\hline 7 & knowledge & 12 & 32 & likeness & 5 \\
\hline 8 & system & 12 & 33 & original & 5 \\
\hline 9 & entities & 12 & 34 & method & 5 \\
\hline 10 & process & 10 & 35 & like & 5 \\
\hline 11 & groups & 9 & 36 & operation & 5 \\
\hline 12 & $\begin{array}{l}\text { arrangemen } \\
t\end{array}$ & 8 & 37 & specific & 5 \\
\hline 13 & grouping & 8 & 38 & human & 5 \\
\hline 14 & items & 8 & 39 & classify & 5 \\
\hline 15 & arranging & 7 & 40 & concepts & 5 \\
\hline 16 & structure & 7 & 41 & unknown & 5 \\
\hline 17 & relations & 7 & 42 & assortment & 5 \\
\hline 18 & scheme & 7 & 43 & work & 5 \\
\hline 19 & attributes & 6 & 44 & principles & 5 \\
\hline 20 & systematic & 6 & 45 & systems & 5 \\
\hline 21 & kinds & 6 & 46 & group & 5 \\
\hline 22 & order & 6 & 47 & criteria & 5 \\
\hline 23 & commom & 6 & 48 & similar & 5 \\
\hline 24 & form & 6 & 49 & hierarchical & 5 \\
\hline
\end{tabular}


Fonte: elaborada pela autora, a partir dos dados coletados.

Observando a Tabela 1, percebe-se que as ocorrências das 50 primeiras palavras, com as frequências de até 5 vezes, são bem representativas para caminhar-se para a análise da palavra classificação.

No Gráfico 1, encontram-se as frequências das primeiras 20 palavras que tiveram até 6 ocorrências na análise léxica das definições sobre classificação.

Gráfico 1 - Palavras mais frequentes nas definições sobre classificação

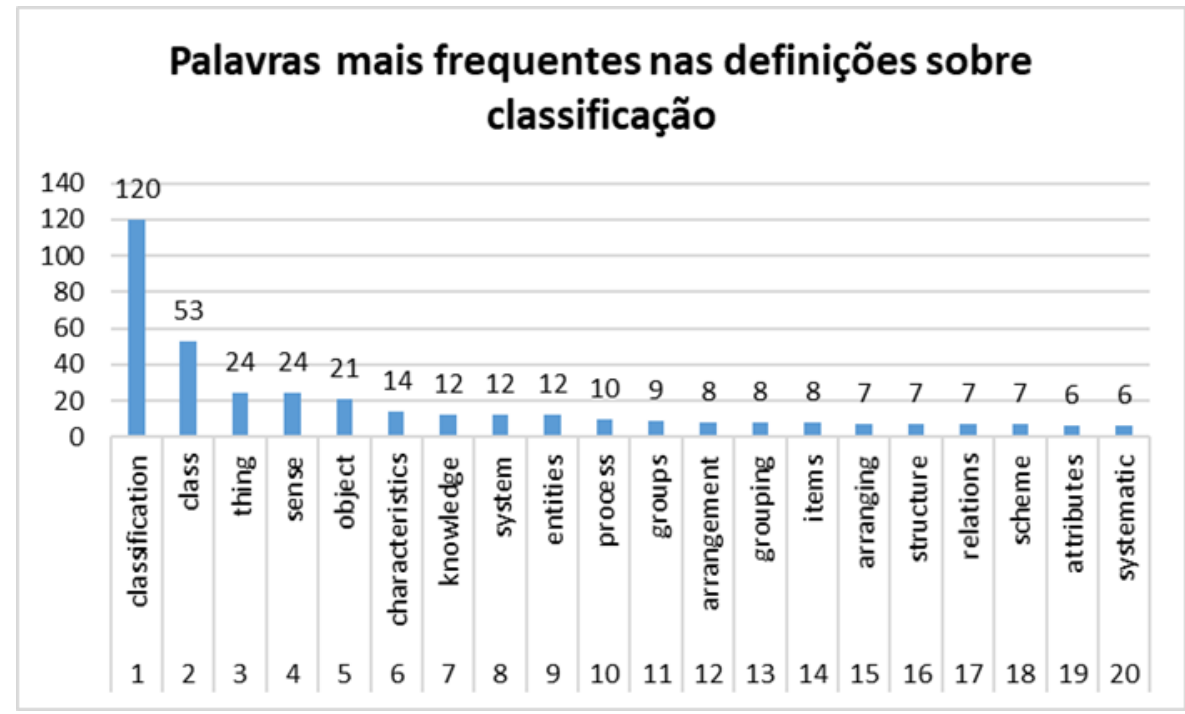

Fonte: elaborada pela autora, a partir dos dados coletados

Em relação ao Gráfico 1, nota-se que a palavra class (classe) aparece em $2^{\circ}$. lugar, e as palavras characteristics (características), knowledge (conhecimento), system (sistema)

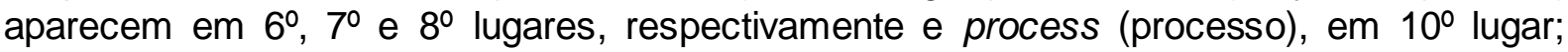
assim, infere-se uma definição para classificação como um processo de dividir o conhecimento (coisa, objeto, entidades e itens) em classes a partir das características comuns para formar-se uma estrutura.

Entre as 20 primeiras palavras colocadas, a classificação, por ser o tema central, aparece, majoritariamente, com a maior frequência. Já a palavra things (coisa), object (objeto), entities (entidades), e items (itens) aparecem respectivamente em $3 .^{\circ}, 5 .^{\circ}, 9 .^{\circ}$ e $14 .^{\circ}$ lugares, sendo utilizadas, muitas vezes, como sinônimos na literatura, demonstrando que não existe um consenso entre os autores, porém, nota-se que thing (coisa) e object (objeto) são as mais utilizadas.

Essa dispersão pode ser ilustrada pela análise da diversidade léxica dessas palavras em relação às palavras classification, thing, object, 
entities e items ${ }^{2}$ por entender que os quatro últimos são utilizados algumas vezes como sinônimos, sendo utilizados na definição da palavra classificação, conforme demostrado na Figura 1.

Figura 1 - Análise da diversidade léxica

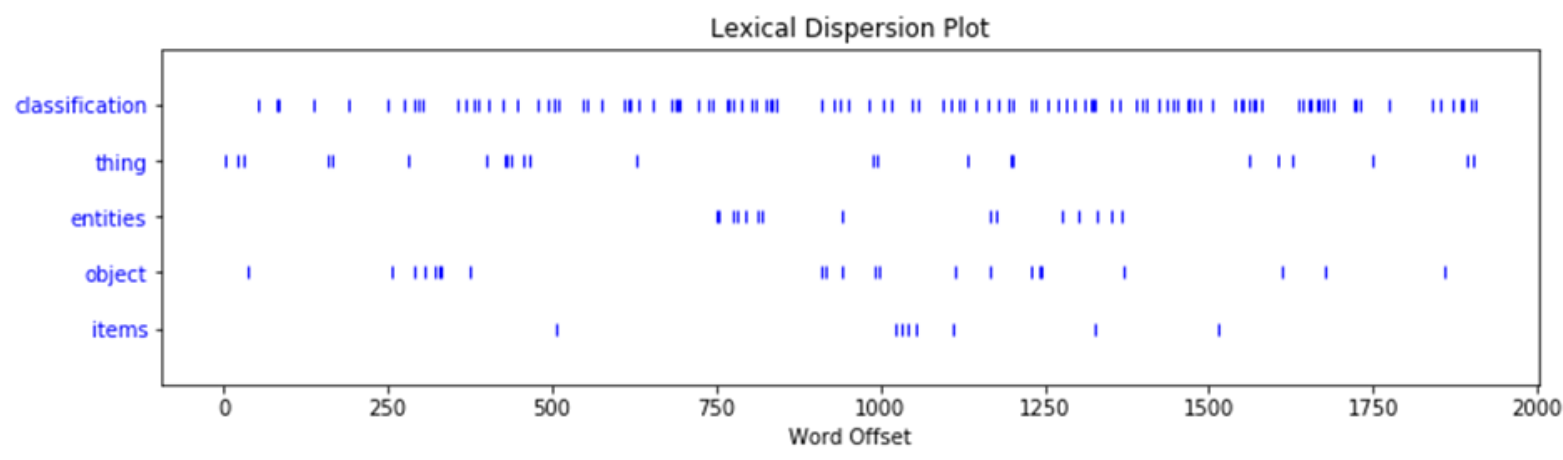

Fonte: elaborada pela autora, a partir dos dados coletados

Ressalta-se, aqui, que a grande ocorrência da palavra classification se deve ao fato de ela representar o tema central de todas as definições. Porém, apesar das palavras thing, object, entities e items estarem entre as quatorze primeiras na frequência, destaca-se que, muitas vezes, essas palavras são utilizadas alternadamente. Essa autora entende que se usa thing e object como entidades concretas, e a palavra entities refere-se a objetos mais abstratos. Pode-se observar pela Figura 1 que o resultado da análise de dispersão demostra que, neste corpus, essas quatro palavras foram utilizadas alternadamente.

Isso também pode ser observado nos bigramas que apareceram pelo menos quatro vezes e no 4-gram, conforme Quadros 1 e 2, a seguir.

Quadro 1- bigramas

\begin{tabular}{ll}
\hline principles criteria & natural classification \\
\hline like thing & classification system \\
classification sense & class classification \\
classification scheme & classification \\
& classification \\
\hline
\end{tabular}

Fonte: elaborada pela autora, a partir dos dados coletados.

\footnotetext{
2 Esta autora esclarece que, na abordagem deste artigo, coisa (thing) é um objeto inanimado distinto de um ser vivo senciente; objeto (object) é uma pessoa ou coisa para a qual uma ação ou sentimento específico é direcionado, material que pode ser visto e tocado; entidade (entities) é algo que tem a existência distinta e independente de uma realidade objetiva e conceitual; itens (items) são partes que compõem alguma coisa, são unidades, elementos.
} 
Quadro 2 - 4-gram

\begin{tabular}{l|l}
\hline $\begin{array}{l}\text { theory, structure, knowledge, relations, 2, } \\
\text { structure, knowledge, relations, different, } \\
2, \\
\text { knowledge, relations, different, parts, 2, }\end{array}$ & $\begin{array}{l}\text { relations, different, parts, knowledge, 2, } \\
\text { hierarchical, series, nested, class, 2, } \\
\text { class, shared, characteristics, perceived, } \\
2,\end{array}$ \\
\hline
\end{tabular}

Fonte: elaborada pela autora, a partir dos dados coletados

Nesta parte da análise léxica, constatou-se que as palavras classification, class e thing são as que aparecem no mesmo contexto nas definições. Ressalta-se que, por ser uma amostra pequena, não houve muitas variáveis nesta similaridade, como pode ser observado no Gráfico 2.

Gráfico 2 - Resultado da análise léxica das palavras classification, class e thing

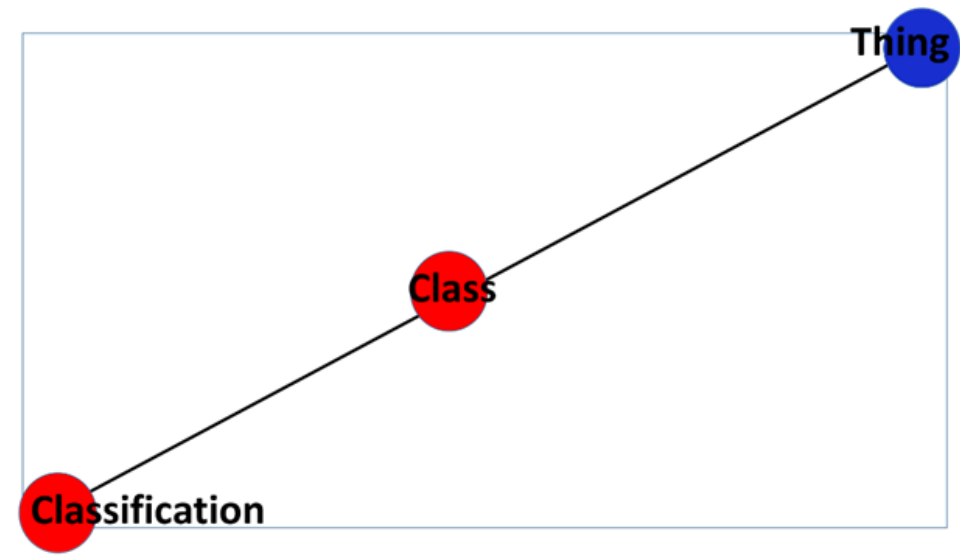

Fonte: elaborado pela autora, a partir dos dados coletados.

Esse processo automático auxiliou, a partir de uma abordagem quantitativa com a verificação das ocorrências principais, obter indicadores importantes para a realização da fase posterior, a análise categorial.

No segundo estágio, a análise categorial, realizou-se a análise temática das definições selecionadas sobre classification, selecionando-se as frases/palavras que apresentavam elementos conceituais significativos como indutoras, e destacaram-se os trechos em que ocorriam essas expressões no contexto da definição. As palavras de maior ocorrência na análise léxica serviram como um indicador para esta análise. Esse procedimento teve como objetivo extrair do corpus os temas relacionados 
ou os elementos conceituais das definições para auxiliar na criação do conjunto de categorias para realização da análise categorial.

A partir desse resultado, passou-se a fazer a codificação para escolha das Unidades de Contexto (UC) e das Unidades de Registro (UR). Para as Unidades de Contexto, foram considerados o tema classificação e as ideias expressas nas definições que constituem o corpus pelas suas características. Para as Unidades de Registros (UR), foram considerados os resultados dessas características, mesmo que elas não estivessem explicitamente nas definições, para realizar a análise categorial. 0 conjunto final das categorias foi composto da seguinte forma:

(a) Unidade de Contexto (UC): abordagens das definições de classificação quanto ao campo de estudo, à natureza, ao contexto, ao processo, ao método, à estrutura, à perspectiva do autor, ao perfil do autor.

(b) Unidades de Registro (UR): Biblioteconomia, Biologia, Botânica, Informática, Filosofia, História, Linguística, Lógica, Classificação filosófica, Classificação bibliográfica, Classificação científica, contexto histórico, contexto científico, contexto interdisciplinar, agrupamento, ordenação método analítico sindético, método dedutivo, método dedutivo/indutivo, método pragmático, estrutura sistemática, estrutura facetada, estrutura conceitual, disciplina/teoria, processo, produto, instrumento, Bibliotecário/Classificador, Biólogo, Botânico, Pesquisador, Filósofo.

A partir desses resultados, partiu-se para a última etapa da técnica, com o tratamento dos resultados, inferência e interpretação, que será apresentada a seguir.

\section{Terceira etapa}

Constituiu-se do tratamento dos resultados, da inferência e da interpretação, com a validação do conjunto de "Categorias" e a apreciação e a análise dos resultados. Para orientar a análise, foram construídos quadros de temas e subtemas que compunham o conteúdo das definições sobre classificação da amostra, e quais perspectivas os autores utilizaram em suas definições. Nesta etapa, a análise teve como objetivo mapear os elementos constitutivos das definições sobre classificação no contexto interdisciplinar da palavra, em relação aos pressupostos e aos objetivos desejados, cujas análises estão expostas a seguir.

\section{Inferências e interpretações dos dados}

Inicialmente, foram analisadas as 40 definições apresentadas sobre classificação propostas por diversos autores, em inglês, em ordem cronológica, publicadas como apêndice do artigo "Classification", de autoria de Birger Hjørland, da revista Knowledge Organization, v. 42, n. 2, 
2017. Como registrado anteriormente, a seleção do corpus baseou-se somente no Apêndice deste artigo. Decidiu-se mantê-las em inglês, sem tradução, para não ter o inconveniente de tirá-las do contexto.

A Tabela 2 apresenta o conjunto de categorias com as ocorrências das temáticas e subtemáticas extraídas do corpus, em uma relação das ocorrências das temáticas/subtemáticas versus números de definições nas quais elas ocorrem.

Tabela 2 - Ocorrências de temáticas e subtemáticas no corpus

\begin{tabular}{|c|c|c|c|}
\hline Temática & \multicolumn{2}{|l|}{ Subtemáticas } & \multirow{2}{*}{$\begin{array}{l}\text { Número da definição } \\
12,14,15,16,17,18,21,22, \\
23,24,26,27,28,29,30,33, \\
34,35,36\end{array}$} \\
\hline \multirow{27}{*}{$\begin{array}{l}\text { As diversa s acepções } \\
\text { da palavra } \\
\text { classificação: }\end{array}$} & \multirow{8}{*}{$\begin{array}{l}\text { Quanto ao } \\
\text { campo de } \\
\text { estudo }\end{array}$} & Biblioteconomia & \\
\hline & & Biologia & $5,9,10,11,20,31$ \\
\hline & & Botânica & 3 \\
\hline & & Informática & 25 \\
\hline & & Filosofia & $1,2,6,13,19$ \\
\hline & & História & 4,7 \\
\hline & & Linguística & $32,37,40$ \\
\hline & & Lógica & $8,38,39$ \\
\hline & \multirow{7}{*}{$\begin{array}{l}\text { Quanto ao } \\
\text { perfil do autor }\end{array}$} & $\begin{array}{l}\text { Bibliotecário/ } \\
\text { Classificador }\end{array}$ & $\begin{array}{l}12,14,15,16,17,18,21,22, \\
23,24,26,27,28,29,34,35,36\end{array}$ \\
\hline & & Biólogo & $5,9,10,11,20,31$ \\
\hline & & Botânico & 3 \\
\hline & & Filósofo & $1,2,6,7,8,13,19$ \\
\hline & & $\begin{array}{l}\text { Fonte de } \\
\text { referência }\end{array}$ & $21,30,37,40$ \\
\hline & & Historiador & 4 \\
\hline & & Pesquisador & $25,32,33,38,39$ \\
\hline & \multirow{3}{*}{$\begin{array}{l}\text { Quanto à } \\
\text { natureza }\end{array}$} & $\begin{array}{l}\text { Classificação } \\
\text { filosófica }\end{array}$ & $1,2,6,8,13$ \\
\hline & & $\begin{array}{l}\text { Classificação } \\
\text { bibliográfica }\end{array}$ & $\begin{array}{l}12,14,15,16,17,18,21,22, \\
23,24,26,27,28,33,34,35, \\
36,37\end{array}$ \\
\hline & & $\begin{array}{l}\text { Classificação } \\
\text { científica }\end{array}$ & $\begin{array}{l}3,4,5,7,9,10,11,19,20,25, \\
29, \\
30,31,32,38,39,40\end{array}$ \\
\hline & \multirow{3}{*}{$\begin{array}{l}\text { Quanto ao } \\
\text { contexto }\end{array}$} & histórico & $4,19,20$ \\
\hline & & científico & $\begin{array}{l}1,2,3,5,6,7,8,9,10,11,13 \\
24,25,26,28,29,30,31\end{array}$ \\
\hline & & interdisciplinar & $\begin{array}{l}12,14,15,16,17,18,21,22, \\
23,27,32,33,34,35,36,37,38, \\
39,40\end{array}$ \\
\hline & \multirow[b]{2}{*}{$\begin{array}{l}\text { Quanto ao } \\
\text { processo }\end{array}$} & agrupamento & $\begin{array}{l}1,2,3,4,6,7,8,9,10,11,13 \\
23,26,30,32,40\end{array}$ \\
\hline & & ordenação & $\begin{array}{l}5,12,14,15,16,17,18,19,20, \\
21,22,24,25,27,28,29,31, \\
33,34,35,36,37,38,39\end{array}$ \\
\hline & \multirow{4}{*}{$\begin{array}{l}\text { Quanto ao } \\
\text { método }\end{array}$} & Analítico sindético & 18 \\
\hline & & dedutivo & $\begin{array}{l}1,2,8,9,11,13,14,15,20,21, \\
22,23,24,26,28,29,30,31 \\
32,33,34,35,37,40\end{array}$ \\
\hline & & $\begin{array}{l}\text { Dedutivo/ } \\
\text { indutivo }\end{array}$ & $3,16,17,19,25,27,36,38,39$ \\
\hline & & pragmático & $4,5,6,7,10,12$ \\
\hline
\end{tabular}




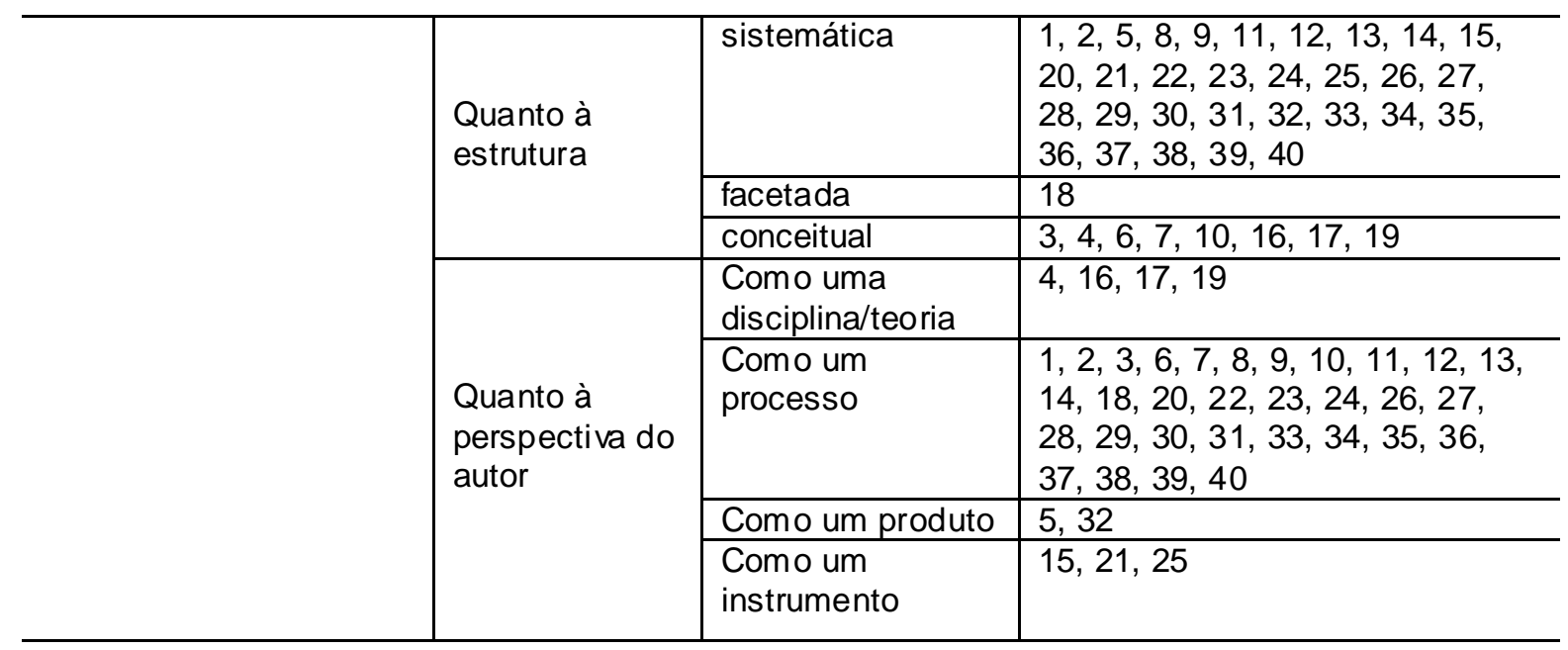

Fonte: elaborada pela autora, a partir dos dados coletados.

Ao observar a Tabela 2, percebe-se que a palavra classificação pode ter diversas acepções, mas algumas características repetem-se na maioria delas. A partir das análises realizadas nas oito subtemáticas levantadas, pode-se mapear as perspectivas que cada autor trouxe na sua visão da palavra classificação. Ressalta-se que, concomitantemente à coleta das temáticas e subtemáticas, foi realizada uma análise específica, considerando a área de conhecimento na qual o autor atuava e, para isso, precisou-se fazer uma pesquisa sobre o perfil do autor em questão, já que as definições não apresentavam essa informação, e considerou-se importante trazer essa informação para a caracterização da autoria.

Apresentam-se a seguir os dados na forma de um gráfico, o que facilita a percepção das incidências das subtemáticas em cada definição de classificação, em relação ao valor absoluto de ocorrências. Assim, a despeito das subtemáticas campo de estudo e perfil do autor, pode-se verificar que as 40 definições foram originadas de oito campos de estudos, e sete perfis autorais diferentes, distribuídas conforme apresentadas nos Gráficos 3 e 4, a seguir.

Gráfico 3 - Características das definições de classificação quanto ao campo de estudo 


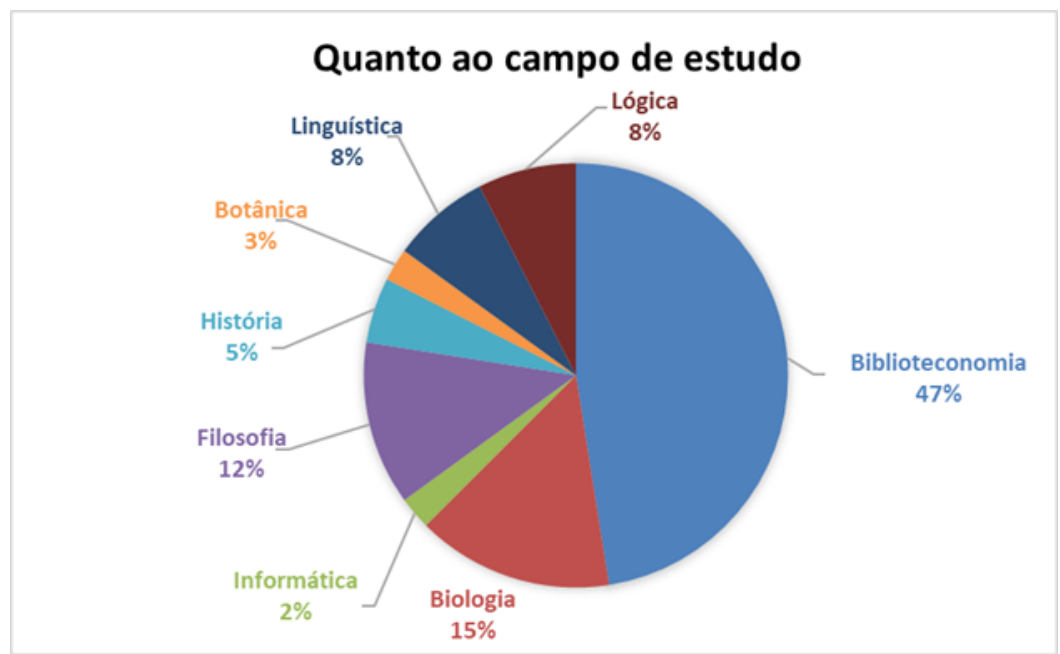

Fonte: elaborado pela autora, a partir dos dados coletados.

No Gráfico 3, observa-se que $47 \%$ das definições foram originadas no âmbito da área da Biblioteconomia, o que não é surpresa, visto que essa área estuda a classificação como área, como processo e como instrumento. Em seguida, aparece a Biologia com 15\%, área em que a classificação foi utilizada para nomear, identificar e classificar os seres vivos, criando um sistema, conhecida como taxonomia de Lineu, e $12 \%$ das definições tiveram como ponto de partida a Filosofia, berço da categorização aristotélica. A Lógica e a Linguística contribuíram com $8 \%$ e, na sequência, a História, com 5\%, a Botânica com 3\%, e a Informática com $2 \%$, ou seja, com apenas uma definição de cada área no corpus representado. O Gráfico 4 confirma a perspectiva do Gráfico 3.

\section{Gráfico 4 - Características das definições de classificação quanto ao perfil dos autores}

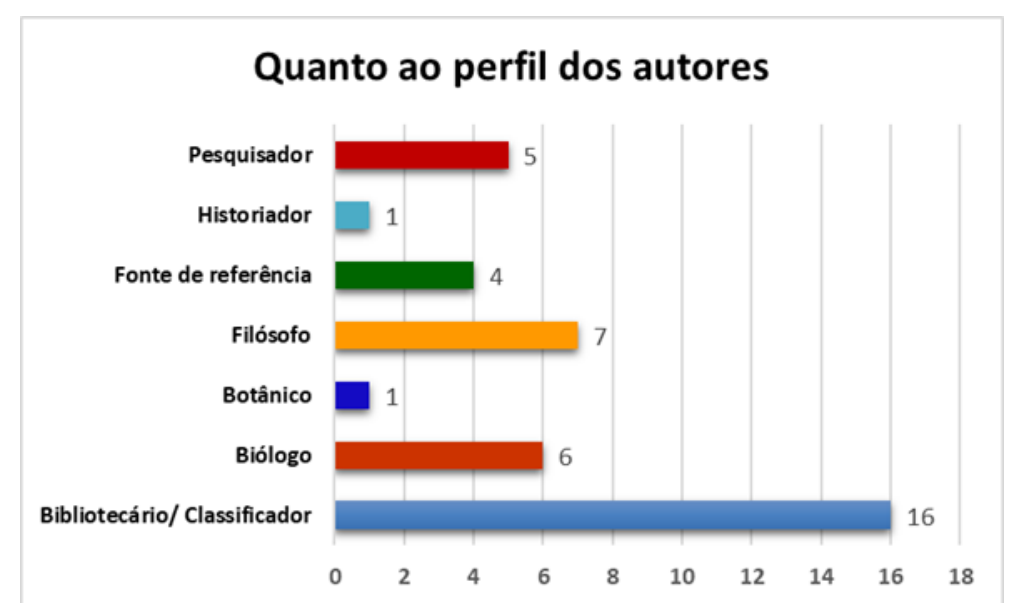

Fonte: elaborado pela autora, a partir dos dados coletados.

No Gráfico 4, registra-se que 16 autores entre os 40 do corpus possuem a formação e/ou trabalham como bibliotecário/indexador; em seguida, aparecem as 7 definições que foram propostas por filósofos; já 
os biólogos contribuíram com 6; os pesquisadores sugeriram 5, que, nesse caso, foram considerados aqueles estudiosos da área; 4 definições foram registradas em fontes de referências, e um botânico e um historiador apresentaram uma cada.

$\mathrm{Na}$ análise da subtemática "Quanto à natureza", inferiu-se que três tipos de classificação podem ser considerados intuitivamente na amostra: a classificação bibliográfica, com ocorrência em 18 definições, perfazendo $45 \%$ do total do corpus; seguida pela classificação científica, com 17 ocorrências, representando 43\%; e, por último, a classificação filosófica, em cinco definições, representando $12 \%$ das ocorrências; conforme ilustrado no Gráfico 5, a seguir.

Gráfico. 5 Características das definições de classificação quanto à natureza

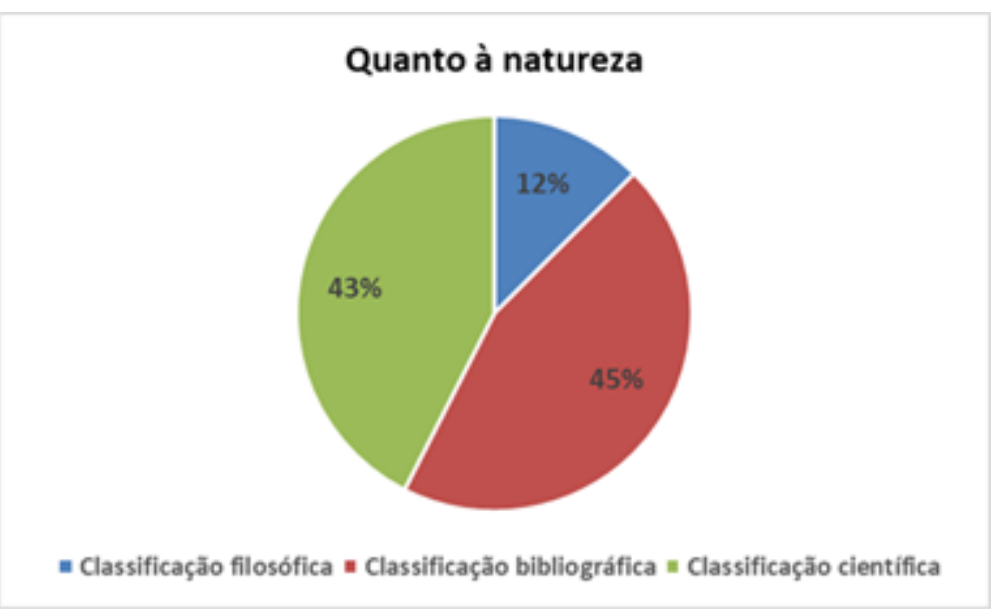

Fonte: elaborado pela autora, a partir dos dados coletados.

Os resultados do Gráfico 5, como os anteriores, confirmam que as áreas da Biblioteconomia e da Ciência da Informação contribuem em maiores ocorrências, até então, com as definições da amostra estudada. $\mathrm{Na}$ análise dessa UC "Quanto à natureza" considerou-se não somente os elementos conceituais significativos como indutores, mas também as UCs "Campo de estudo" e "Perfil dos autores".

Em relação a quais contextos as definições foram sugeridas, pode-se vislumbrar, conforme o Gráfico 6, uma maior incidência no contexto interdisciplinar com 19 ocorrências, representando 48\% das definições, seguido pelo contexto científico com 18 ocorrências, $45 \%$, e aparecem apenas 3 definições sugeridas dentro do contexto histórico, ou seja, 7\%.

Gráfico 6 - Características das definições de classificação quanto ao contexto 


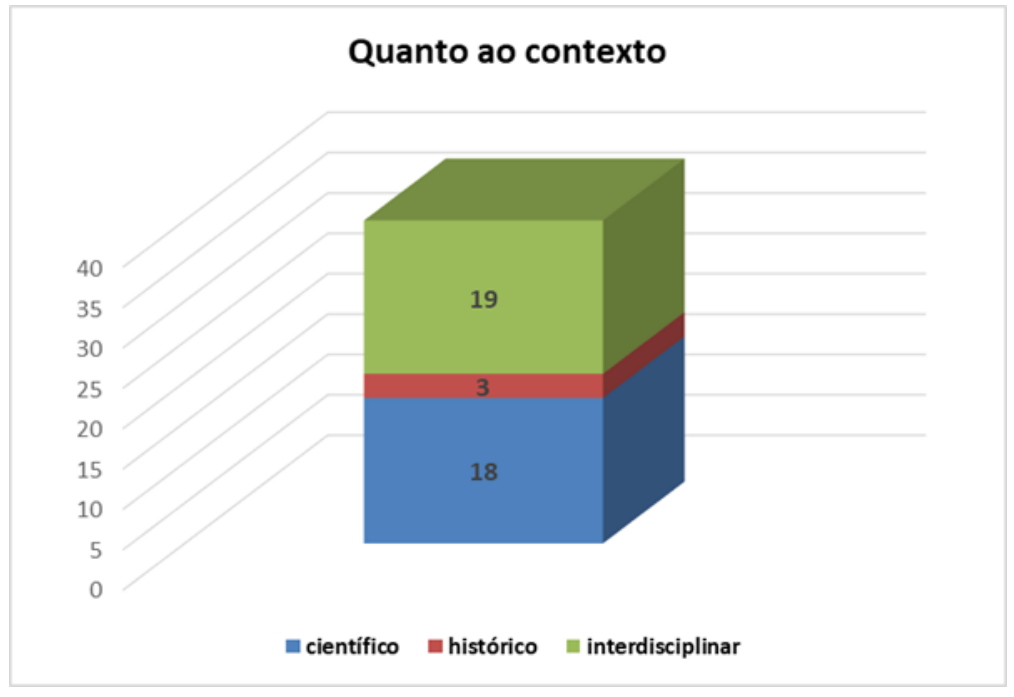

Fonte: elaborado pela autora, a partir dos dados coletados.

Para observar em qual contexto determinada definição estava inserida, considerou-se as circunstâncias nas quais ela foi elaborada, como, por exemplo: se fazia menção a algum princípio do historicismo, no caso do contexto histórico. O contexto científico baseou-se naquelas definições que tinham como embasamento o estudo científico mais específico do conhecimento, e, no caso do interdisciplinar, aquelas definições que tratavam do conhecimento de um modo geral, de caráter universal.

O Gráfico 7 representa os processos que foram evidenciados nas definições. Nesse caso, emergiram-se dois processos principais: o agrupamento e a ordenação. As definições que tinham uma perspectiva categorial aristotélica, de análise e síntese, com formação de agrupamento por características a partir de critérios, foram consideradas na UR Agrupamento, enquanto as definições que tinham a perspectiva a partir da ordem das classes, com a distribuição de elementos segundo as características, foram consideradas na UR Ordenamento.

O que se nota no Gráfico 7, a seguir, é que as definições com perspectivas de agrupamento são em menor número, representando $40 \%$ do total, sendo que apenas 16 das 40 definições evidenciaram em seu enunciado o objetivo de definir e hierarquizar o conhecimento. No que tange à UR ordenamento, interpretou-se que em $60 \%$, ou seja, em 24 definições, ocorriam esta perspectiva, esta abordagem de um conjunto hierárquico finito de gêneros e espécies, que funciona por dicotomias sucessivas, correspondendo como as áreas de Biblioteconomia e da Ciência da Informação abordam as definições.

Gráfico 7 - Características das definições de classificação quanto ao processo 


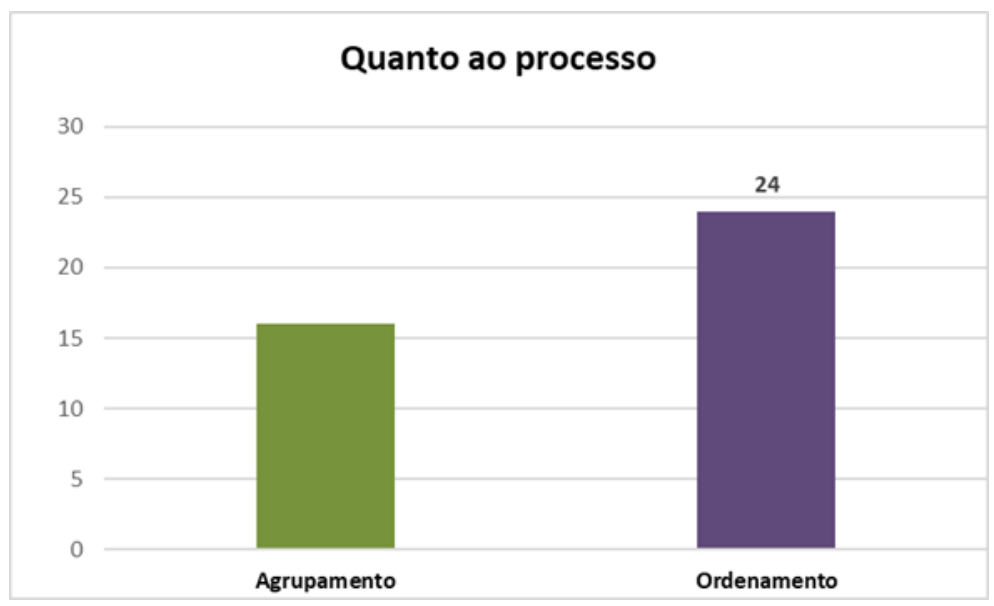

Fonte: elaborado pela autora, a partir dos dados coletados.

$\mathrm{Na}$ análise da UC "Método", no Gráfico 8, revelou-se a predominância do método dedutivo, representando $60 \%$ do total das características de métodos sugeridos nas definições. Em seguida, aparece o método dedutivo/indutivo, o qual foi mencionado nas definições que sugerem que a classificação deva ser construída indutivamente, a partir de unidades específicas de informação, para, posteriormente, agrupá-las em grandes classes, representando $23 \%$ da amostra.

Gráfico 8 - Características das definições de classificação quanto ao método

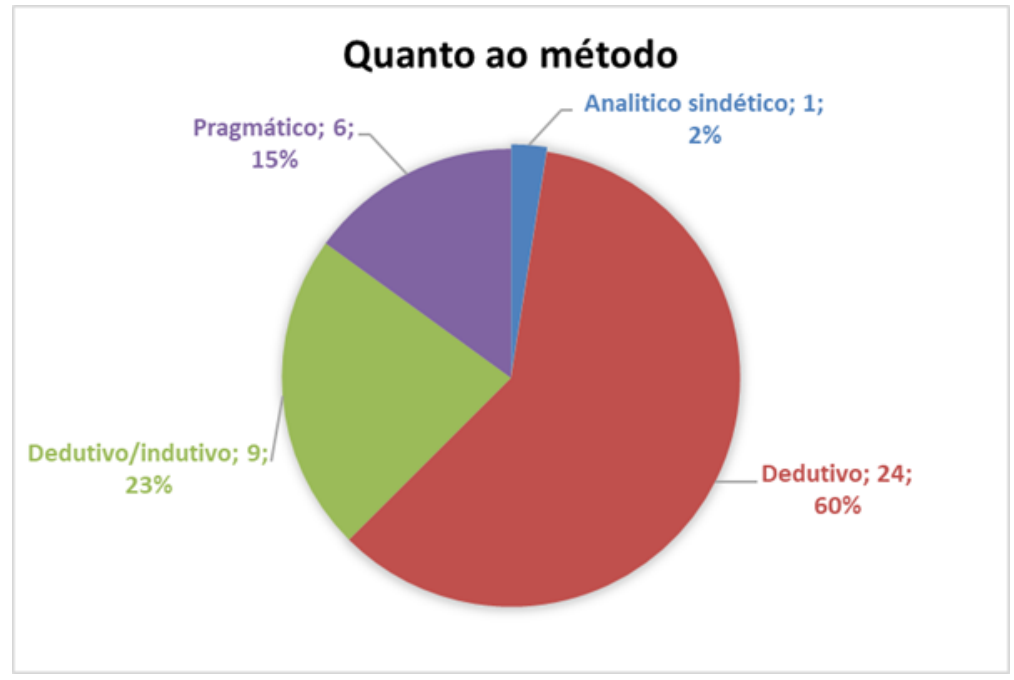

Fonte: elaborado pela autora, a partir dos dados coletados

O método pragmático foi percebido em $15 \%$ da amostra, sendo somente em 6 definições e, por fim, o método analítico sindético, com $2 \%$, sendo apenas nas definições sugeridas por Ranganathan (1967).

O Gráfico 9 nos revela as três estruturas que foram sugeridas na amostra das 40 definições. Como já era de se esperar, a estrutura sistemática foi a mais mencionada, ocorrendo em $78 \%$ da amostra, ou 
seja, em 31 definições da amostra. Na sequência, têm-se a estrutura conceitual com $20 \%$ e a estrutura facetada com $2 \%$ do total.

Gráfico 9 - Características das definições de classificação quanto à estrutura

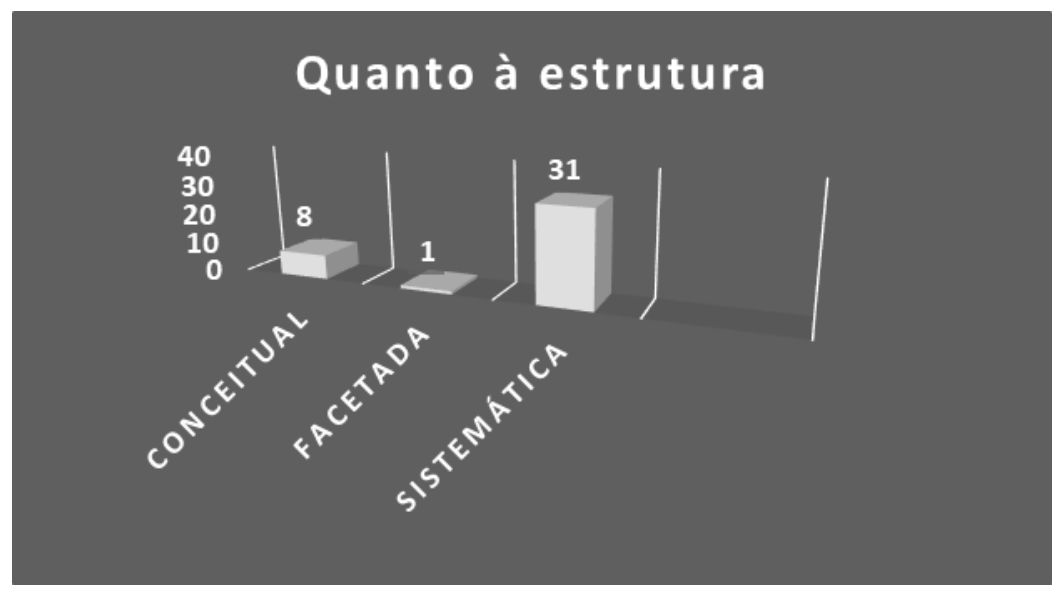

Fonte: elaborado pela autora, a partir dos dados coletados

Este percentual alto em relação à estrutura sistemática se deve à característica inerente da abordagem sistemática dos saberes dentro do contexto da história das classificações das ciências. Já a estrutura facetada teve somente $2 \%$ de ocorrência, sendo mencionada na definição sugerida por Ranganathan, conforme observado, também no Gráfico 9.

O Gráfico 10, último desta análise, traz um dos aspectos mais discutidos em relação às diversas acepções que a palavra classificação pode ter. Isso porque é comum ver na literatura, conforme mencionado anteriormente, autores definindo-a como uma área do conhecimento; outros, como processo; e alguns outros, como um sistema de classificação. Costuma-se, também, encontrar autores que trazem essas três abordagens em suas explicações.

Gráfico 10 - Características das definições de classificação quanto à perspectiva do autor 


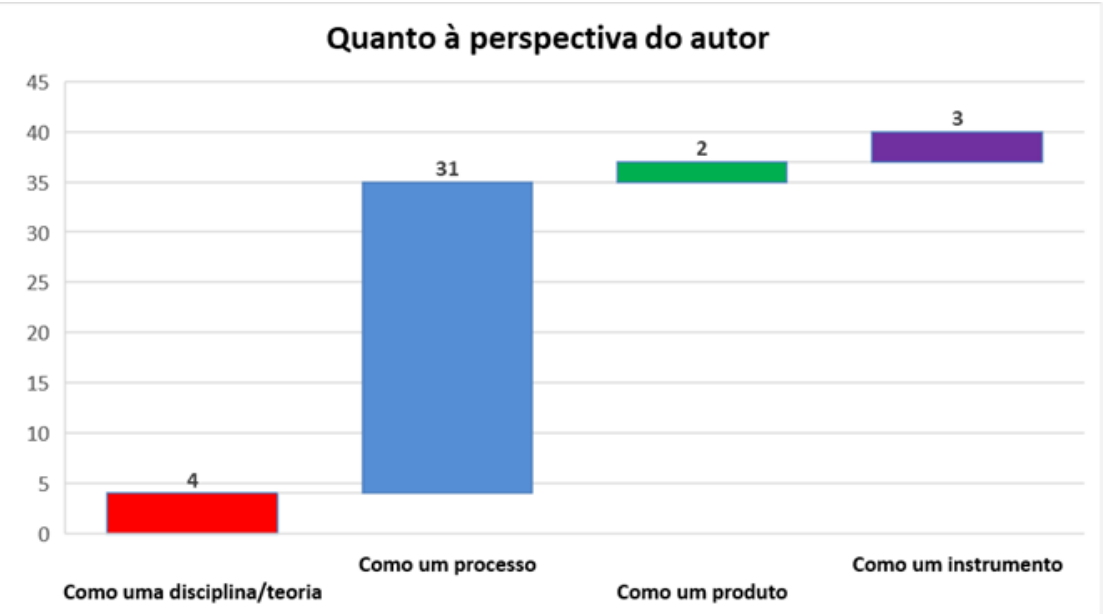

Fonte: elaborado pela autora, a partir dos dados coletados

No Gráfico 10, pode-se notar, no entanto, que a maioria das definições da amostra apresentam a classificação como um processo, com 31 ocorrências, ou seja, em $77 \%$ do total; posteriormente aparece $10 \%$ dos autores que a definem como uma disciplina/teoria; como um instrumento de classificação, $8 \%$ dos autores e, como um produto, a estrutura que resulta do processo, apenas $5 \%$ da amostra.

\section{Considerações finais}

Este artigo trouxe resultados parciais de reflexões realizadas no âmbito do projeto de pesquisa PQ-CNPq sobre "Estudo sobre o estatuto teórico metodológico da análise de assunto", que tem como objetivo principal estudar os fundamentos teóricos metodológicos da análise de assunto, verificando, principalmente, a evolução metodológica e quais são as contribuições de pesquisas que visam melhorar a subjetividade desse processo e contribuir para o ensino e a aprendizagem da disciplina Análise de Assunto na graduação.

Neste caso específico, a partir da leitura do artigo "Classification" (HJØRLAND, 2017), suscitaram-se questões sobre a importância do papel do processo de análise de assunto para a classificação. Neste artigo, esta resposta não foi trazida, porém, começa-se a desenhar esta perspectiva a partir do estudo sobre as diversas acepções que a palavra classificação pode ter na literatura para, assim, delinear um viés mais alinhado à pesquisa referenciada.

O resultado da análise realizada nas diversas definições da palavra classificação veio confirmar os pressupostos apontados na metodologia deste artigo, no entanto percebe-se que, apesar de possuir diversas acepções, algumas características repetem-se na maioria delas.

A análise léxica apontou que as palavras things (coisa), object (objeto), entities (entidades), e items (itens) aparecem alternadamente nas definições, muitas vezes, como sinônimos na literatura, demonstrando 
que não existe um consenso entre os autores, porém, nota-se que thing (coisa) e object (objeto) são as mais utilizadas. Isso demonstra a dispersão da diversidade léxica dessas palavras, sendo que as palavras classification, class e thing são as que aparecem no mesmo contexto nas definições.

Após a análise categorial, observou-se que a maioria das definições foram originadas no âmbito do campo da Biblioteconomia e Ciência da Informação e na Biologia, apesar de terem seu embasamento teórico na filosofia. Notou-se, também, que a maioria dos autores são bibliotecários/indexadores; seguidos de filósofos e biólogos. Evidenciouse, também, uma predominância acentuada das características nas análises das definições, como classificação bibliográfica, dentro de um contexto científico, com o uso do processo de ordenamento e do método dedutivo, organizada na estrutura sistemática, e a maioria dos autores definiu a classificação como um processo. Em síntese, a análise das características das definições a partir das maiores ocorrências na representatividade do corpus pode ser concluída conforme representado no Quadro 4, a seguir.

Quadro 4 - Resumo das características com maiores ocorrências nas definições.

\begin{tabular}{|l|l|l|l|l|l|l|l|l|}
\hline UC & Área de estudo & Perfil do Autor & Natureza & Contexto & Processo & Método & Estrutura & Visão do autor \\
\hline UR & Biblioteconomia & $\begin{array}{l}\text { Bibliotecário/ } \\
\text { Classificador }\end{array}$ & $\begin{array}{l}\text { Classificação } \\
\text { bibliográfica }\end{array}$ & Interdisciplinar & Ordenamento & Dedutivo & Sistemática & Como um processo \\
\hline
\end{tabular}

Fonte: elaborada pela autora, a partir dos dados coletados.

Isso posto, confirmou-se que a classificação é objeto central de pesquisas em muitas ciências, visto que a origem partiu dos estudos filosóficos; mais tarde, durante a década de 1940, começam a ficar mais evidentes os estudos sobre a taxonomia biológica e, na matemática, com a intenção de agrupamento. Além dessas áreas, outros campos também estão envolvidos, de uma forma ou de outra, em estudos sobre a classificação como na Antropologia (por exemplo, sistemas parentais, etnolinguísticas) e também em Biologia (Botânica e Zoologia (Linnaeus)), bem como na Linguística e na Ciência da Computação. Desse jeito, o caráter interdisciplinar da palavra classificação é justificável no que tange ao seu uso em múltiplos contextos, mas sempre remete a um processo sistemático, com características lógicas para subdivisão, realizado de acordo com um conjunto de princípios estabelecidos que orientam a estrutura e o relacionamento dos conceitos em uma sequência de classes - sendo essa sua principal característica.

Confirmou-se, também, que a base teórica do processo classificatório sempre remete às classificações filosóficas com os estudos de Platão e Aristóteles. No âmbito da Biblioteconomia e Ciência da 
Informação, a classificação pode ser vista como ciência, como um produto, resultado do processo de divisão, e como a ferramenta utilizada para realizar o processo de classificação.

Conclui-se que a classificação traça a sua história pelas bases categoriais aristotélicas. Começou seu desenvolvimento dicotômico com os estudos de Porfírio, ganhou projeção com o sistema de categorias taxonômica de Carlos Lineu, com a ideia de "divisão e denominação" para classificar os seres em grupos, com a obra A Origem das espécies de C. R. Darwin; e, no âmbito da Biblioteconomia e Ciência da Informação, com os estudos iniciados pelo bibliotecário E. C. Richards (1860-1939), no Século XIX, influenciando os estudos de autores como W. C. B. Sayers (18811960), H. E. Bliss (1870-1955) e S. R. Ranganathan (1892-1972), entre outros. Na área da Biblioteconomia e Ciência da Informação, a classificação tem sido estudada como uma disciplina, um processo de agrupar e ordenar o conhecimento, e como instrumento de representação da informação, contribuindo para a sua organização e recuperação.

\section{Agradecimentos}

Agradeço ao apoio do Conselho Nacional de Desenvolvimento Científico e Tecnológico (CNPq/Brasil), pela concessão da bolsa de Produtividade de Pesquisa (PQ-1D).

\section{Referências}

BACON, Francis. The Advancement of Learning. Auckland: The Floating Press, 2010.

BARDIN, Laurence. Análise de conteúdo. Lisboa: Edições 70, 1977.

BARDIN, Laurence. Análise de conteúdo. Lisboa: Edições 70, 2006.

BARDIN, Laurence. Análise de conteúdo. São Paulo: Edições 70, 2011.

BATLEY, Sue. Classification in theory and practice. Oxford, UK: Chandos, 2005

BERELSON, Bernard.; LAZARSFELD, Paul. Felix. The analysis of communication content. [n.p.] 1948.

BLISS, Henry. E. The Organization of Knowledge and the System of the Sciences. New York, NY: Henry Holt and Company, 1929. 
BOWKER, Geoffrey. C.; STAR, Susan. Leigh. Sorting Things Out: classification its consequences. Cambridge, Mass.: MIT Press, 1999.

CRESWELL, John. W. Projeto de pesquisa: métodos qualitativo, quantitativo e misto. 2. ed. Tradução de Luciana de Oliveira da Rocha. Porto Alegre: Artmed, 2007.

DAHLBERG, Ingetraut. O futuro das linguagens de indexação. Tradução do inglês por Henry B. Cox. Palestra apresentada à Conferência Brasileira de Classificação Bibliográfica, Rio de Janeiro, 12-17 de setembro de 1972. Anais [...] Brasília, IBICT/ABDF, 1979. v. 1, p. 323-334.

DAHLBERG, Ingetraut. Fundamentos teóricos-conceituais da classificação. Revista de Biblioteconomia, Brasília, v. 6, n. 1, jan./jun. p. 9-21, 1978.

DAHLBERG, Ingetraut. International Society for Knowledge Organization (ISKO). In: Encyclopedia of Library and Information Sciences, $3^{\text {rd }}$ ed. New York: Taylor and Francis, 2010.Disponível em:

https://www.taylorfrancis.com/chapters/edit/10.1081/E-ELIS3$120044729 /$ international-society-knowledge-organization-isko-ingetrautdahlberg. Acesso em: 13 mar. 2021.

DAHLBERG, Ingetraut. Teoria do conceito. Ciência da informação, v. 7, n. 2, p. 101-107, 1978.

DOUSA, Thomas. M. The simple and the complex in E. C. Richardson's theory of classification. Observations on an early $\mathrm{KO}$ model of the relationship between ontology and epistemology. In: GNOL, C; MAZZOCCHI, F. (Eds.). Paradigms and conceptual systems in knowledge organization: Proceedings of the Eleventh International ISKO Conference, Rome, February 2010. Würzburg: ERGON VERLAG, 2010. p. 15-22. (Advances in Knowledge Organization, 12).

FOSKET, Douglas J. Systems theory and its relevance to documentary classification. International Classification, v.7. n. 1, 1980. p. 2-5

GARCIA MARCO, Francisco. J.; ESTEBAN NAVARRO, Miguel. A. On some contributions of the cognitive sciences and epistemology to a theory of classification. Knowledge of Information, v. 20, n. 3, p. 126-132, 1993.

HJØRLAND, Birger. Classification. Knowledge Organization, v. 44, n.2, p. 97-128, 2017.

HULME, Edward. W. Principles of Book Classification. A.A.L. Reprints 1. London: The Association of Assistant Librarians. 1950. 
KORNER, Stephan. Classification theory. International Classification, v.3, n.1, p. 3-6, 1976.

LANGRIDGE, Derek W. Classificação: abordagem para estudantes de biblioteconomia. Rio de Janeiro: Interciência, 1977. 120 p.

LANGRIDGE, Derek W. Subject analysis: principles and procedures. London: Bowker-Saur, 1989.

McKENNA, Malcolm.C.; BELL Susan K. Classification of Mammals Above the Species Level. New York, NY: Columbia University Press, 1998.

MINAYO, Maria Cecilia. S. (Org.). Pesquisa social: teoria, método e criatividade. Rio de Janeiro: Vozes, 2001.

PIEDADE, Maria. Antonieta. R. Introdução à teoria da classificação. Rio de Janeiro: Interciência, 1983.

RANGANATHAN, Shiyali Ramamrita. Philosophy of Library Classification. Bangalore: Sarada Ranganathan Endowment for Library Science, 1973a. $133 \mathrm{p}$.

RANGANATHAN, Shiyali. Ramamrita. Classification and Comunication. Bangalore: Sarada Ranganathan Endowment for Library Science, 1973b. 291 p.

RANGANATHAN, Shiyali. Ramamrita. Prolegomena to Library Classification. 3rd ed. London: Asia Publishing House, 1967.

REITZ, Joan. M. Dictionary for library and information science. London: Libraries Unlimited, 2004. p 224.

RICHARDSON, Ernest.C. Classification: theoretical and practical. New York: H.W. Wilson, 1930.

SATIJA, Mohinder P. Classification: some fundamentals, some myths, some realities. Knowledge Organization, v.25, n.1-2, p.32-35, 1998.

SAYERS, Berwick W.C. An Introduction to Library Classification: theoretical, historical, and practical, with readings, exercises, and examination papers. New York: Grafton, 1950.

SHERA, Jesse. H.; EGAN, Margareth. E. The classified catalog, with a code for the construction and maintenance of the classified catalogs. Chicago: American Library Association, 1956. 
SHERA, Jesse. H. Libraries and the Organization of Knowledge. Hamden, CT: Archon Books. 1965.

SMIRAGLIA, Richard. P. The Progress of Theory in Knowledge

Organization. Library Trends, v. 50, n. 3, Winter, p. 330-349, 2002.

SOPER, Mary Ellen et al. The Librarian's Thesauri's. Chicago: ALA, 1990.

SVENONIOUS, Elaine. Ranganathan and classification science. Libri, v. 42, n. 3, p. 176-183, 1992.

VICKERY, Brian. C. Classificação e Indexação nas Ciências. Rio de Janeiro: BNG/Brasilart, 1980.

\section{ANEXO 1 - Definições sobre classificação}

\begin{tabular}{|c|c|}
\hline 1- Plato (c.370 BC) & $\begin{array}{l}\text { "Socrates: That of dividing things again by classes, where the natural joints are, and not } \\
\text { trying to break any part, after the manner of a bad carver." (Plato c. } 370 \text { BC, Phaedrus } \\
265 \mathrm{e}) \text {. }\end{array}$ \\
\hline $\begin{array}{l}\text { 2- Aristotle (350 BCE, Book } \\
\text { I, chap. 1, part 5) }\end{array}$ & $\begin{array}{l}\text { "A'genus' is what is predicated in the category of essence of a number of things } \\
\text { exhibiting differences in kind. We should treat as predicates in the category of essence } \\
\text { all such things as it would be appropriate to mention in reply to the question, 'What is the } \\
\text { object before you?'; as, for example, in the case of man, if asked that question, it is } \\
\text { appropriate to say 'He is an animal'" (Aristotle } 350 \text { BCE, Book I, chap. 1, part 5). Note: "The } \\
\text { logic of classifications, which remains, in this time, the Aristotelian logic, receives } \\
\text { practically no new development until the 18th century" (Parrochia 2016; electronic source, } \\
\text { no pages; see also Sutcliffe 1993). }\end{array}$ \\
\hline $\begin{array}{l}\text { 3- Michel Adanson (1763, } \\
\text { clvi) }\end{array}$ & $\begin{array}{l}\text { "...all parts and qualities, or properties and faculties of plants... barring not even one" } \\
\text { ought to be considered before attempting a classification. }\end{array}$ \\
\hline $\begin{array}{l}\text { 4- David Cranz (1767, vol. } \\
1 \text {, ix) }\end{array}$ & $\begin{array}{l}\text { "I have described what belongs to this science, not according to the classifications [Ger. } \\
\text { Eintheilungen] and characteristics, which are generally adopted by, and needful for the } \\
\text { modern naturalists, but according to a certain affinity or likeness." }\end{array}$ \\
\hline $\begin{array}{l}\text { 5- Carl Linnaeus }(1767, p . \\
152)\end{array}$ & $\begin{array}{l}\text { "Natura Insectorum per plures eorum ætates jam perspecta, superest ut systematice } \\
\text { eadem contemplemur. Recta autem eorum CLASSIFIC ATIO vitam huic scientiæ \& } \\
\text { facultatem conciliat, ubi singula insecta suum quasi nomen ipsa produnt" = "The nature of } \\
\text { insects through their several ages [stages of life] having already been examined, it } \\
\text { remains to contemplate them systematically. Now the correct CLASSIFICATION of them } \\
\text { [sci., insects] furnishes life and means to this science, where individual insects } \\
\text { themselves produce, as it were, their own name." (majuscule in original). }\end{array}$ \\
\hline $\begin{array}{l}\text { 6- Thomas Reid (1785, } \\
\text { p.191) }\end{array}$ & $\begin{array}{l}\text { "Our ability to distinguish and give names to the different attributes belonging to a } \\
\text { single thing goes along with an ability to observe that many things have certain } \\
\text { attributes in common while they differ in others. This enables us to put the countless } \\
\text { hordes of individuals into a limited number of classes, which are called 'kinds' and 'sorts' } \\
\text { - and in the scholastic language called 'genera' and 'species"' (here quoted from Frické } \\
2012,25) \text {. }\end{array}$ \\
\hline $\begin{array}{l}\text { 7- William Whewell }(1840, \\
\text { vol. 1, xxxiii, XC V) }\end{array}$ & $\begin{array}{l}\text { "The attempts at Natural Classification are of three sorts; according as they are made by } \\
\text { the process of blind trial, of general comparison, or of subordination of characters. The } \\
\text { process of Blind Trial professes to make its classes by attention to all the characters, but } \\
\text { without proceeding methodically. The process of General Comparison professes to } \\
\text { enumerate all the characters, and forms its classes by the majority. Neither of these } \\
\text { methods can really be carried into effect. The method of Subordination of Characters } \\
\text { considers some characters as more important than others; and this method gives more } \\
\text { consistent results than the others. This method, however, does not depend upon the } \\
\text { Idea of Likeness only, but introduces the Idea of Organization or Function" (italics in } \\
\text { original). }\end{array}$ \\
\hline - Jol & $\begin{array}{l}\text { "Classification, thus regarded, is a contrivance for the best possible ordering of the id } \\
\text { of objects in our minds; for causing the ideas to accompany or succeed one anoth }\end{array}$ \\
\hline
\end{tabular}




\begin{tabular}{|c|c|}
\hline 300) & $\begin{array}{l}\text { such a way as shall give us the greatest command over our knowledge already acquired, } \\
\text { and lead more directly to the acquisition of more. The general problem of Classification, } \\
\text { in reference to these purposes, may be stated as follows: To provide that things shall be } \\
\text { thought of in such groups, and those groups in such an order, as will best conduce to the } \\
\text { remembrance and to the ascertainment of their laws." }\end{array}$ \\
\hline $\begin{array}{l}\text { 9- William Benjamin } \\
\text { Carpenter (1847, I. §2) }\end{array}$ & $\begin{array}{l}\text { "The object of all Classification ... [is] to bring together those beings which most } \\
\text { resemble each other and to separate those that differ." }\end{array}$ \\
\hline $\begin{array}{l}\text { 10- Charles Danwin (1859, } \\
\text { p.420) }\end{array}$ & $\begin{array}{l}\text { "... all true classification is genealogical ..." (See also Mayr and Bock 2002; Padian } \\
\text { 1999.). }\end{array}$ \\
\hline $\begin{array}{l}\text { 11- Thomas Henry Huxley } \\
(1869, \text { p. } 1)\end{array}$ & $\begin{array}{l}\text { "By the classification of any series of objects is meant the actual, or ideal, arrangement } \\
\text { together of those which are like and the separation of those which are unlike, the } \\
\text { purpose of this arrangement being to facilitate the operation of the mind in clearly } \\
\text { conceiving and retaining in the memory, the characters of the objects in question." }\end{array}$ \\
\hline $\begin{array}{l}\text { 12-Charles Ammi Cutter } \\
(1876, \text { p.10) }\end{array}$ & "Class, a collection of objects having characteristics in common". \\
\hline $\begin{array}{l}\text { 13- Robert Adamson (1901, } \\
\text { v.1, p.185) }\end{array}$ & $\begin{array}{l}\text { "The process of arranging the objects of some province of experience into kinds or } \\
\text { groups, characterized by the possession of common marks. As ordinarily defined, it } \\
\text { involves more than logical DIVISION (q.v.), the rules of which furnish the minimal } \\
\text { conditions of the process. In addition, classification takes into account (1) either the } \\
\text { specific purpose of the arrangement, or (2) the natural conjunctions of marks which } \\
\text { are of most importance. In either case, the aim of classification is to render possible the } \\
\text { greatest number of general propositions regarding the objects, and so to facilitate the } \\
\text { complete and systematic survey of them. The ideal of a classification that is not } \\
\text { determined by special, human ends, as e.g. in classification of occupations in a census } \\
\text { return, is to copy in its systematic arrangement the real order of interdependence in } \\
\text { the things themselves. What is called 'artificial,' as opposed to natural classification, } \\
\text { differs in degree only, not in kind. Literature: MILL, Logic, Bk. IV. chaps. vii, viii; VENN, } \\
\text { Empirical Logic, chap. xxx; JEVONS, Princ. of Sci., chap. Xxx. (R.A.)." }\end{array}$ \\
\hline $\begin{array}{l}\text { 14- Ernest Cushing } \\
\text { Richards on }(1901, p .1)\end{array}$ & $\begin{array}{l}\text { Classification is the "putting together of like things, or more fully described, it is the } \\
\text { arranging of things according to likeness and unlikeness. It may also be expressed as } \\
\text { the sorting and grouping of things. It is convenient sometimes, to speak of 'likeness and } \\
\text { unlikeness' but really in classification it is 'likeness' which rules while 'unlikeness' is } \\
\text { merely what is left over when likeness has been defined. The 'putting together of like } \\
\text { things' is therefore the fullest and most exact form of the definition." }\end{array}$ \\
\hline $\begin{array}{l}\text { 15- Henry E. Bliss (1935, } \\
\text { p.3) }\end{array}$ & $\begin{array}{l}\text { "In dealing with the multiplicity of particular things, actualities, and specific kinds, we } \\
\text { find that some are alike, in general characters and in specific characteristics; and we } \\
\text { may consequently relate them in a class, or classes, that is classify them." }\end{array}$ \\
\hline $\begin{array}{l}\text { 16- Jason E. L. Farradane } \\
(1950, \text { p.83,p. } 87)(1952, p . \\
73-74)\end{array}$ & $\begin{array}{l}\text { "Classification is a theory of the structure of knowledge, i.e. of the relations between } \\
\text { different parts of knowledge. No arbitrary method of grouping, however carefully } \\
\text { applied, is true classification. The problem is primarily epistemological. What is true } \\
\text { knowledge, and what are true relations between the parts of knowledge? It is } \\
\text { essential to define these if the classification is to be true and logically sound." "A } \\
\text { classification indicates the relations between items of knowledge." "A classification } \\
\text { represents a theory of the structure of knowledge, i.e. of the relations between the } \\
\text { different parts of knowledge. The basic problem is to determine what these relations } \\
\text { are and how they link the different concepts from our knowledge into a coherent } \\
\text { structure. The arbitrary or 'deductive' subdivision of an assumed total of knowledge } \\
\text { cannot give a true representation of these relations, which do not consist only of } \\
\text { groupings of a class and its members, or division of a whole into its parts. It was } \\
\text { shown that a classification must be constructed 'inductively', or upwards, piecing } \\
\text { together known fragments of relations." }\end{array}$ \\
\hline $\begin{array}{l}\text { 17- Jesse H. Shera }(1965 \text {, } \\
\text { p. } 120, \text { p. 127) }\end{array}$ & $\begin{array}{l}\text { "Classification is the crystallization or formalizing of inferential thinking, born of } \\
\text { sensory perception, conditioned by the operation of the human brain, and shaped by } \\
\text { human experience. It lies at the foundation of all thought, but it is pragmatic and it is } \\
\text { instrumental." "He [the librarian] must appreciate classification, not as a tool, but as a } \\
\text { discipline in which is to be studied the reaction and response of a living mind to the } \\
\text { record left by a distant and usually unknown mind; a discipline that seeks to achieve a } \\
\text { better understanding of the changing patterns of thought and the points of contact at } \\
\text { which they can be related to specific units of recorded information." }\end{array}$ \\
\hline $\begin{array}{l}\text { 18- Shiyali Ramam rita } \\
\text { Ranganathan }(1967,\end{array}$ & $\begin{array}{l}\text { Ranganathan distinguished five senses of 'classification'. 1. "Division. (See Chap CC). } \\
\text { This is the primitive meaning of the term 'classification'. Even a child practises }\end{array}$ \\
\hline
\end{tabular}




\begin{tabular}{|c|c|}
\hline Chapter CP, 77-78) & 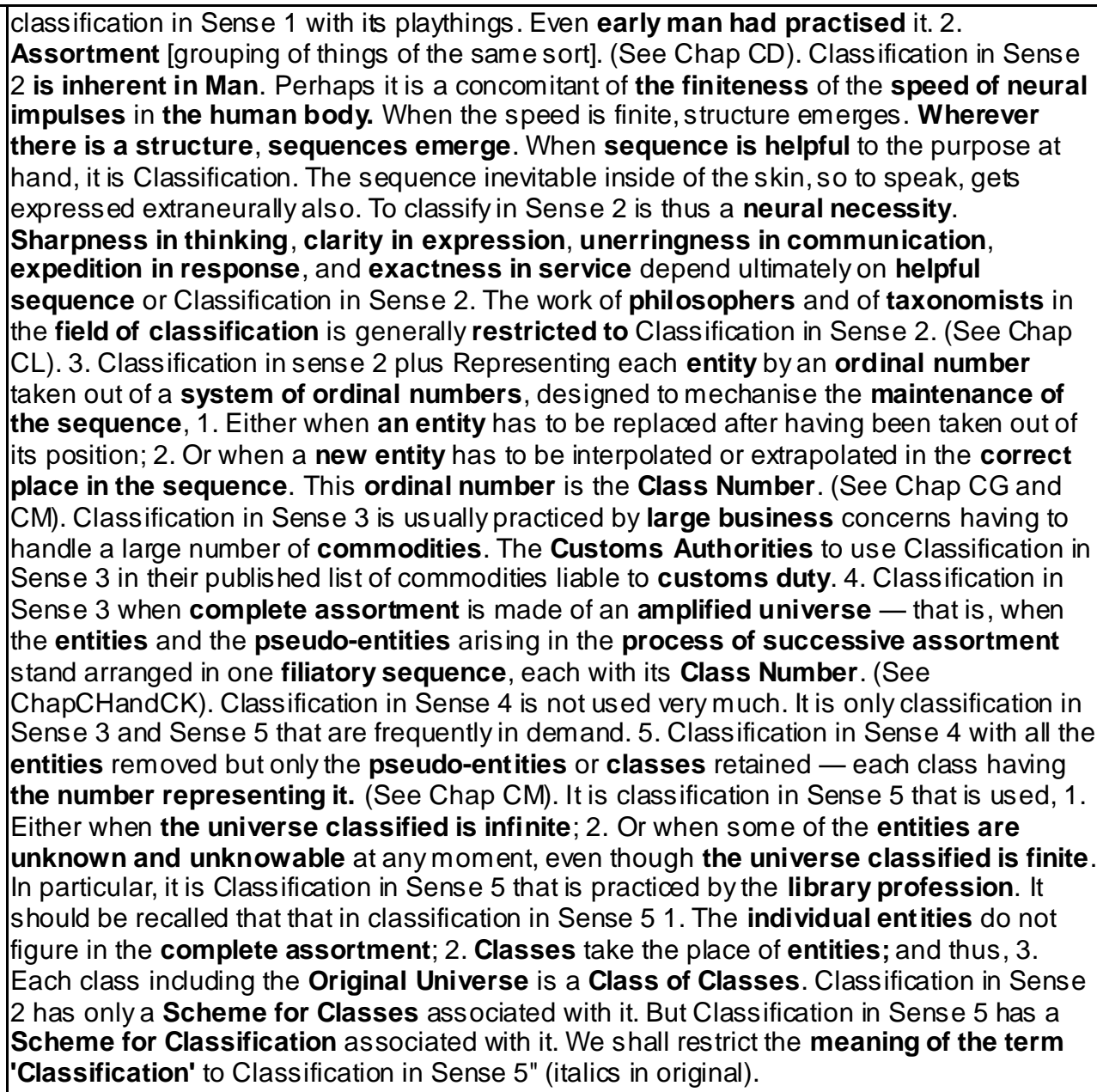 \\
\hline $\begin{array}{l}\text { 19- Bonifatii Mikhailovich } \\
\text { Kedrow (1975, 1:3 } \\
\text { emphasis original) }\end{array}$ & $\begin{array}{l}\text { "Klassifikation. Verfahren zur Unterteilung eine Klasse K von Dingen usw. in Teilklassen" } \\
\text { (628). "Die dialektisch-materialistische Einstellung zur Klassifikation besteht also nicht etwa } \\
\text { darin, dass sie im Gegensatz zur antidialektischen die Berechtigung und den Wert von } \\
\text { Klassifikationen bestritte, sondern darin, dass sie die These von der zeitlichen und } \\
\text { strukturellen Relativität der Klassifikationen vertritt, während die antidialektische Auffassung } \\
\text { die Klassifikationen in jeder Hinsicht als absolut betrachtet." This can perhaps be } \\
\text { generalized in this way: Classification of objects means the display of connections } \\
\text { between the objects in a certain order or in a system reflecting certain basic principles. } \\
\text { Kedrow found that the principle of historicism must govern all natural classifications } \\
\text { (see section 4.2cy Genetic/historicist approaches to classification). }\end{array}$ \\
\hline $\begin{array}{l}\text { 20- Phillip R. Sloan (1981, } \\
\text { p. 68) }\end{array}$ & $\begin{array}{l}\text { "The arrangement of objects or entities into groups or classes, usually on the basis of } \\
\text { perceived similarity and difference." }\end{array}$ \\
\hline $\begin{array}{l}\text { 21- ISO 5127-6 (1988, p. } \\
93)\end{array}$ & $\begin{array}{l}\text { A classification system is an "indexing language intended for a structured } \\
\text { representation of documents or data, through the use of indexes and corresponding } \\
\text { terms, in order to allow systematic access, resorting to an alphabetical index, if } \\
\text { necessary" (here cited from Simões et al. 2016,531; note that this standard has been } \\
\text { revised by ISO 5127:2001). }\end{array}$ \\
\hline $\begin{array}{l}\text { 22- Lois Mai Chan }(1994, \mathrm{p} . \\
\text { 259) }\end{array}$ & $\begin{array}{l}\text { Classification is "the multistage process of deciding on a property or characteristic of } \\
\text { interest, distinguishing things or objects that possesses that property from those } \\
\text { which lack it, and grouping things or objects that have the property or characteristic in } \\
\text { common into a class. Other essential aspects of classification are establishing } \\
\text { relationships among classes and making distinctions within classes to arrive at } \\
\text { subclasses and finer divisions." }\end{array}$ \\
\hline $\begin{array}{l}\text { 23- Francis L. Miksa (1994, } \\
\text { p. 144) }\end{array}$ & $\begin{array}{l}\text { "[Bibliographic] Classification is the activity of creating categories into which } \\
\text { bibliographic items of all kinds may be placed (i.e., the work of the classificationist) and } \\
\text { also the activity of ident ifying bibliographical items in terms of the categories already } \\
\text { extant in a given system (i.e., the work of the classifier). It encompasses systems for } \\
\text { arranging items on the shelves of libraries (sometimes called 'bibliothecal' } \\
\text { classification), as well as systems for arranging the surrogates of items in catalogs }\end{array}$ \\
\hline
\end{tabular}




\begin{tabular}{|c|c|}
\hline & $\begin{array}{l}\text { (sometimes called 'bibliographical' classification). It includes classificatory systems } \\
\text { based on all kinds of item characteristics (subject, form, author, citation, size, etc.), in all } \\
\text { forms of order (logical and s ystematic, alphabetical, faceted, etc.), with all kinds of } \\
\text { operating methods (pre- and post-coordinated, statistically based clustering and } \\
\text { identification, etc.), and differing in scope from the universal to the very narrow. Finally, } \\
\text { library classification embraces a wide range of purposes, although most often its chief } \\
\text { purpose has been to facilitate document retrieval." }\end{array}$ \\
\hline $\begin{array}{l}\text { 24- Frederick Wilfrid } \\
\text { Lancaster (1998, p. 17) }\end{array}$ & $\begin{array}{l}\text { Classification is "sorting items into 'conceptual classes'" and "forming classes of } \\
\text { objects on the basis of their subject matter". }\end{array}$ \\
\hline $\begin{array}{l}\text { 25- Geoffrey Bowker and } \\
\text { Susan Leigh Star }(1999, \mathrm{p} . \\
\text { 10) }\end{array}$ & $\begin{array}{l}\text { "Aclassification is a spatial, temporal, or spatio-temporal segmentation of the world. A } \\
\text { 'classification system' is a set of boxes (metaphorical or literal) into which things can be } \\
\text { placed to perform some kind of work - bureaucratic or knowledge production." }\end{array}$ \\
\hline $\begin{array}{l}\text { 26- Arlene G. Taylor (1999, } \\
\text { p. 237) }\end{array}$ & $\begin{array}{l}\text { "The placing of subjects into categories; in organizing of information, classification is } \\
\text { the process of determining where an information package fits into a given hierarchy } \\
\text { and then assigning the notation associated with the appropriate le vel of the hierarchy } \\
\text { to the information package and its surrogate." }\end{array}$ \\
\hline 27- Satija (2000, p. 222) & $\begin{array}{l}\text { "Classification means to divide objects/entities (both abstract and concrete) on the } \\
\text { bas is of their differences or, conversely, the grouping of entities on the basis of their } \\
\text { similarities. Classification is any process of dividing, sorting, grouping, arranging, } \\
\text { ordering, ranking, mapping and correlating." }\end{array}$ \\
\hline $\begin{array}{l}\text { 28- Elaine Svenonius } \\
(2000, \text { p. } 10)\end{array}$ & $\begin{array}{l}\text { "Organization can take many forms. Its prototypical form is classification. Classification } \\
\text { brings like things together. In traditional classifications, like things are brought } \\
\text { together with respect to one or more specified attributes. Any number of attributes can } \\
\text { be used to form classes of documents embodying information, such as same size or } \\
\text { color, same subject, or same author. However, the most important attribute for a system } \\
\text { whose objectives is to organize information is the attribute of 'embodying the same } \\
\text { work'. " }\end{array}$ \\
\hline $\begin{array}{l}\text { 29- Hubert Feger (2001, } \\
1966)\end{array}$ & $\begin{array}{l}\text { "Classification is the assignment of objects to classes". Later on, this was expanded } \\
\text { (Feger } 2015,805) \text { : "The fundamental goal of classification is to find structures common } \\
\text { to a group of objects, using properties to classify the objects into subgroups based } \\
\text { on the similarity of their properties" }\end{array}$ \\
\hline $\begin{array}{l}30 \text { - The Portuguese } \\
\text { Language Dictionary of the } \\
\text { Academy of Sciences } \\
\text { (Academia das Ciências de } \\
\text { Lis boa } 2001,837 \text { cited in } \\
\text { Simões et al. } 2016,531 \text { ) }\end{array}$ & $\begin{array}{l}\text { Classification is the "action of distributing in classes, by categories ... according to } \\
\text { precise criteria" }\end{array}$ \\
\hline $\begin{array}{l}\text { 31- Ernst Mayr and Walter } \\
\text { Joseph Bock (2002, p.191) }\end{array}$ & $\begin{array}{l}\text { "The logical consequence of the definition of class is that classification must be defined } \\
\text { as the ordering of diversity into classes of similar entities. And this has been } \\
\text { traditionally the almost universally accepted concept of classification.... Aclassification } \\
\text { of organisms is bas ed on the shared possession of their diverse attributes. The units } \\
\text { of similarity in a Darwinian classification are called taxonomic characters that have the } \\
\text { property of being homologous to one another in the several entities or groups. The } \\
\text { claim of a few modem } \\
\text { authors that there is no agreement on the definition of the word "classification" is } \\
\text { quite misleading. Actually, prior to } 1950 \text { there was virtually total unanimity on the usage } \\
\text { (in classification) of the words classification and class, as referring to the grouping of } \\
\text { similar items. A classification is defined as "The arrangement of entities in a } \\
\text { hierarchical series of nested classes, in which similar or related classes at one } \\
\text { hierarchical level are combined comprehensively into more inclusive classes at the } \\
\text { next higher le vel" (176). "Class - (in classification) A group or collection of entities } \\
\text { (individuals), possessing attributes or traits in common ('being similar'), a kind or sort, } \\
\text { grouped together under a general or class name. Classification - The arrangement of } \\
\text { similar entities (objects) in a hierarchical series of nested classes, in which each more } \\
\text { inclusive higher-level class is subdivided comprehensively into less inclusive classes at } \\
\text { the next lower level. Darwinian classification - The ordered grouping of organisms } \\
\text { into classes, according to their similarities and consistent with their inferred } \\
\text { evolutionary history. Downward classification - Establishing groups by logical } \\
\text { division. Evolutionary classification - A classification that duly considers both } \\
\text { evolutionary processes, the ecological adaptiveness of evolutionary divergence } \\
\text { (degree of difference) and the genealogy (phylogeny) of the taxa. Basically equal to a } \\
\text { Darwinian classification. Hierarchical classification - The system of ranks that } \\
\text { indicates the categorical level (level of difference) of each taxon" (191). }\end{array}$ \\
\hline
\end{tabular}


32- Kjeld Schmidt and Ina Wagner (2004, p. 392)

33- Dagobert Soergel (2004, p.358)

34- Faria and Pericão (2008, p.258)

35- Clare Beghtol (2010, p.1045)

36- Ingetraut Dahlberg

37- Oxford English Dictionary (2010)

39- Daniel Parrochia and Pierre Neuville (2013, p.21)

38- Adriano Veloso and Wagner Meira (2011, p. 9) (2010, p. 2941)

"Classification, in turn, is a special practice of categorization, involving pre-established and systematic systems of signs. That is, classification is a linguistic operation of applying a classification scheme, i.e., an ordered set of signs that is pre-established according to (a) some general principles and criteria of ordering and (b) some procedures of identification and naming. In short, an act of classification is an application of a classification scheme. Classification systems (such as thesauri) can thus be seen as instantiations of classification schemes. [...] Classifications and categorizations are both convention-based practices and equally so. But classifications are convention-based in a quite specific sense. In the case of categorization there are no pre-established principles and criteria for determining the correctness of an act of categorization. With acts of classification, however, such pre-established principles and criteria exist, in that they specify relationships between items in terms of, for example, class/ membership, part/whole, composition, cause/effect, origin/fate, function, ownership, value/risk, location, or state. Accordingly, an actor applying a classification scheme in a particular case can be held accountable in terms of the principles, criteria, and procedures of the classification scheme."

"Aclassification is a structure that organizes concepts into a meaningful hierarchy, possibly in a scheme of facets. The classification of living things is a taxonomy. (The term taxonomy is increasingly used for any type of classification.) A classification is now often called an ontology, particularly if it gives richer concept relationships" (bold in original omitted here).

Classification is a "group of ordered concepts, distributed systematically in classes, forming a structure" and a "structuring of concepts into classes and subdivisions to express the existing semantic relationships between them" (here cited from Simões et al. 2016, 531).

"To classify means to put things into meaningful groups. Things can be physical objects, ideas, events, or anything else that human beings can perceive or imagine, and a meaningful group can be formed using any characteristic or combination of characteristics of the things. Groups can be considered to be pemanent or they can be considered temporary responses to a need of the moment."

"With this joumal [International classification, 1974-1992, thereafter Knowledge organization], 'classification' was unders tood as a multi-meaning word that includes the following concepts: 1 . classification in the sense of 'classification system', i.e., a system of classes arranged in hierarchical or faceted order; 2 . classification in the sense of classifying, i.e., establishing a system of classes; 3 , classification in the sense of classing, i.e., relating the classes of a classification system to objects or subjects of reality; and 4. classification in the sense of classification science, i.e., relating to this field of study and its activities" (list typography added).

"classification, n. Origin: Aborrowing from Latin. Etymon: Latin classificatio. Etymology: < post-classical Latin classificatio (1673 in a Geman source; 1767 in Linnaeus) > classical Latin classis class n. + -ficātiōn-, -ficātiō suffix. Compare German Klassifikation (1760 or earlier as †Classification), Swedish klassifikation (1740 as †classification), Danish klassifikation (1748), French classification (1780), Italian classificazione (1796). Compare slightly later classify v. The result of classifying; a systematic distribution, allocation, or arrangement of things in a number of distinct classes, according to shared characteristics or perceived or deduced affinities. Also: a system or method for classifying. 1. The action of classifying or arranging in classes, according to shared characteristics or perceived affinities; assignment to an appropriate class or classes. 2. A category to which something is assigned; a class."

"In a classification problem, there is a set of input-output pairs (also referred to as instances or examples) of the form $\mathrm{zi}=(\mathrm{xi}$; $\mathrm{yi})$ : Each input $\mathrm{xi}$ is a fixed-length record of the form $<a 1, \ldots$, al $;$; where each ai is an attribute-value. Each output yi draws its value from a discuirrete and finite set of possibilities $y=\{c 1, \ldots, c p\}$, and indicates the class to which zi belongs. Cases where yi $=$ ? indicate that the correct class of zi is unknown. There is a fixed but unknown conditional probability distribution $P(y \mid x)$, that is, the relationship between inputs and outputs is fixed but unknown" "This formulation implies that the classification problem corresponds to the problem of function approximation."

"Definition 1.9.1 We call 'classification' the operation consisting of sharing, distributing or allocating objects in classes or groups which are, in general, less numerous than them. [10] This is also the result of this operation. We want, as much as it is possible, this result to be constant, i.e. the classification must remain stable for a little transformation [11] of data". 
40- WordNet 3.1 (2016-0521)

"defines four senses of the noun 'classification' of which three are relevant for this entry: 1) "S: (n) categorization, categorisation, classification, compartmentalization, compartmentalisation, assortment (the act of distributing things into classes or categories of the same type) 2) S: (n) classification, categorization, categorisation (a group of people or things arranged by class or category) 3) (n) classification, categorization, categorisation, sorting (the basic cognitive process of arranging into classes or categories)".

Fonte: HJØRLAND, 2017, p. 122 